\title{
Ascending Price Vickrey Auctions for General Valuations
}

\section{Citation}

Mishra, Debasis, and David C. Parkes. 2007. Ascending price Vickrey auctions for general valuations. Journal of Economic Theory 132(1): 335-366.

\section{Published Version}

doi:10.1016/j.jet.2005.09.004

\section{Permanent link}

http://nrs.harvard.edu/urn-3:HUL.InstRepos:3996850

\section{Terms of Use}

This article was downloaded from Harvard University's DASH repository, and is made available under the terms and conditions applicable to Other Posted Material, as set forth at http:// nrs.harvard.edu/urn-3:HUL.InstRepos:dash.current.terms-of-use\#LAA

\section{Share Your Story}

The Harvard community has made this article openly available.

Please share how this access benefits you. Submit a story.

\section{Accessibility}




\title{
Ascending Price Vickrey Auctions For General Valuations*
}

\author{
Debasis Mishra ${ }^{\dagger}$ David C. Parkes ${ }^{\ddagger}$ \\ First version: February 2004, This version: July 2005
}

\begin{abstract}
Ascending price auctions typically involve a single price path with buyers paying their final bid price. Using this traditional definition, no ascending price auction can achieve the Vickrey-Clarke-Groves (VCG) outcome for general private valuations in the combinatorial auction setting. We relax this definition by allowing discounts to buyers from the final price of the auction (or alternatively, calculating the discounts dynamically during the auction) while still maintaining a single price path. Using a notion called universal competitive equilibrium prices, shown to be necessary and sufficient to achieve the VCG outcome using ascending price auctions, we define a broad class of ascending price combinatorial auctions in which truthful bidding by buyers is an ex post Nash equilibrium. Any auction in this class achieves the VCG outcome and ex post efficiency for general valuations. We define two specific auctions in this class by generalizing two known auctions in the literature [11, 24].
\end{abstract}

Keywords: combinatorial auctions; multi-item auctions; primal-dual algorithm; universal competitive equilibrium; Vickrey auctions

JEL Classification Numbers: D44, D50, C62

${ }^{*}$ The second author is supported in part by NSF grant IIS-0238147. We thank Sven de Vries, Veronika Grimm, Sébastien Lahaie, Rudolf Müller, Lyle Ungar, Rakesh Vohra, and two anonymous referees for valuable feedback on the paper. We also thank seminar participants at the CORE Mathematical Programming seminar, INFORMS 2004 annual meeting, Maastricht University, and TU Münich for their feedback on this research. Some of the results in Section 3.1 of this paper appeared as a brief announcement in the Proceedings of Fifth ACM Conference on Electronic Commerce [20].

${ }^{\dagger}$ Center for Operations Research and Econometrics, Université Catholique de Louvain, Belgium, mishra@core.ucl.ac.be

${ }^{\ddagger}$ Corresponding author. Maxwell Dworkin 229, DEAS, Harvard University, 33 Oxford Street, Cambridge, MA - 02138, Email:parkes@eecs.harvard .edu, Phone: (617)-384-8130, Fax: (617)-495-9837 


\section{Introduction}

Ascending price auctions are preferred over their sealed-bid counterparts in practical settings $[10,9,26]$. In the context of selling a single item, the ascending price English auction shares the economic efficiency of the sealed-bid second-price Vickrey auction [31] for private value models.

The sealed-bid Vickrey-Clarke-Groves (VCG) mechanism [31, 8, 15] generalizes the Vickrey auction to combinatorial auctions $[29,30]$ with multiple items and general (private) non-additive valuations and retains its ex post efficiency and dominant-strategy incentivecompatibility properties. Taken together, the economic properties of the VCG mechanism and the practical benefits of an ascending price auction have generated interest in designing efficient ascending price combinatorial auctions, achieving the outcome of the VCG mechanism. Several papers have addressed this issue for restricted valuation domains: for the one-to-one assignment problem by Demange et al. [13], for homogeneous items with nonincreasing marginal valuations by Ausubel [2], for heterogeneous valuations with gross substitutes valuations by Ausubel and Milgrom [3] and de Vries et al. [11].

For a general private valuations model, i.e., with no externalities and free disposal but no other restrictions on valuations (such as requirements that items are substitutes of each other), there is a negative result due to de Vries et al. [11]. They show that gross substitutes valuations are almost the largest valuation domain for which an ascending price auction can achieve the VCG outcome. Of course, this negative result depends on how an ascending price auction is defined. These authors adopted a definition in which the auction should have a single price path and the buyers should pay the final price in this price path. Unlike an earlier definition of an ascending price auction, due to Gul and Stacchetti [16], they did not restrict prices to item prices but allowed a non-anonymous (i.e., personalized for every buyer) and non-linear (i.e., non-additive over items) price path.

To overcome this impossibility, one needs to relax the definition of an ascending price auction. One possible relaxation is to allow multiple price paths in an auction ${ }^{1}$. Still in the restricted case of gross substitutes valuations, Ausubel [1] maintains multiple price paths and is able to maintain simple anonymous and linear prices for each price path. Combining information from all price paths, Ausubel's auction includes an incremental protocol which determines VCG payments of buyers upon termination. This idea is further generalized by de Vries et al. [11], who show that their auction can be run multiple times, once for every buyer, to calculate the VCG payments of buyers for general valuations.

The use of multiple price paths in these auctions requires each buyer to bid on price paths which are only used to calculate payment of a specific buyer and serve no other purpose. This is not appealing in practice because buyers have no incentive to participate in such price paths. Besides, introducing multiple price paths creates overhead for buyers to bid in

\footnotetext{
${ }^{1} \mathrm{~A}$ price path is a sequence of increasing or decreasing prices. Multiple price path means multiple such sequences. These price paths may be run simultaneously or sequentially one after the other.
} 
an auction. It also has less transparency and simplicity than a single price path auction. For this reason, the following question merits research and is the focus of this paper:

Is there a relaxation of the traditional definition of ascending price auctions which maintains a single price path and still terminates with the VCG outcome for general valuation profiles in an ex post Nash equilibrium?

In pursuit of an answer to this question, we explore an alternative relaxation of the definition of ascending price auctions in de Vries et al. [11] that maintains a single price path. We allow the final payments made by each buyer to differ from the final clearing prices. In fact, this relaxation is already present in Ausubel' auction [1], in addition to the use of multiple price paths. In our characterization, the final payments made by each buyer can be determined either as a one-time discount from the final clearing prices or dynamically during the auction as in Ausubel's earlier auctions [1, 2]. We believe that the use of a single price path together with incremental discounting makes for transparent and simple combinatorial auctions. In the presence of such discounting, prices in our auctions act as means of eliciting preferences only and not as prices which are paid eventually by buyers.

Our main contribution is a broad class of ascending price auctions which achieve the VCG outcome for general valuations using a single price path. For this, we introduce the concept of universal competitive equilibrium (UCE) prices. UCE prices are competitive equilibrium (CE) prices (possibly non-linear and non-anonymous) of the main economy as well as $\mathrm{CE}$ prices of every "marginal economy" (an economy where a single buyer is excluded). We show that UCE prices are necessary and sufficient to achieve the VCG outcome using an ascending price auction. Our broad class of ascending price auctions search for UCE prices, with VCG payments determined either as one-time discounts from the clearing prices or dynamically during the auction. The overall discount to a buyer from the final clearing price on his bundle of items is his marginal contribution to the revenue of the seller at the final auction prices. Truthful bidding is an ex post Nash equilibrium in such auctions.

Even though discounts are given, buyers still respond to auction prices, sans discount, for bidding. Thus, the prices in our auctions act as means for eliciting preferences of buyers. The actual payments of buyers are functions of the auction prices but not always the prices they see. As pointed out by de Vries et al. [11], it is not possible to design an ascending price auction achieving VCG outcome which maintains a single price path and in which buyers pay what they see as prices. For exactly the same reason, the price path generated by subtracting the discounts from our auction prices in each round is not ascending.

The general class of auctions is described as a "black-box model" in which prices are maintained in each round and buyers can report their demand set at current prices as bids. The auctions increase prices in each round, maintaining quasi-CE prices for the main economy and marginal economies and making progress towards UCE prices. We present two specific auctions within this class. One of them generalizes the primal-dual auction in de Vries et al. [11] and the other generalizes the $i$ Bundle auction in Parkes and Ungar [27] (known to 
implement a subgradient algorithm $[11,12])$. The beauty of the latter auction is the simple and transparent price adjustment step. But, auctions based on primal-dual algorithms are believed to have faster convergence properties [11]. In both cases, we present the first ascending (multi-item) Vickrey auction for general valuations with a single price path. Instead of giving discounts to buyers at the end of our auctions, we can also dynamically calculate their discounts during the auction.

For the special case of buyers-are-substitutes, known to be necessary and sufficient for the existence of CE prices that simultaneously give VCG payoffs to each buyer [7], UCE prices are achieved as soon as CE prices of the main economy are achieved and no additional rounds of bidding are required to determine final payments. In comparison, the auctions in de Vries et al. [11] and Ausubel and Milgrom [3] need a stronger condition to achieve the VCG outcome because they do not allow discounts upon termination.

The rest of the paper is organized as follows. In Section 2, we introduce the concept of UCE price and give its connection to the VCG mechanism. In Section 3, we define our broad class of auctions. We give two specific auctions and analyze their theoretical properties in Sections 4 and 5. We summarize and conclude in Section 6.

\section{Universal Competitive Equilibrium}

We define the combinatorial allocation problem and the concept of universal competitive equilibrium (UCE) price. Later, we will illustrate how this UCE price concept can be used to design ascending price auctions.

\subsection{The Model}

A seller has $n$ heterogeneous indivisible items to sell. The set of items is denoted by $A=$ $\{1, \ldots, n\}$. There are $m(\geq 2)$ buyers, denoted by $B=\{1, \ldots, m\}$. The set of all bundles of items is denoted by $\Omega=\{S \subseteq A\}$. Naturally, $\emptyset \in \Omega$. For every buyer $i \in B$ and every bundle $S \in \Omega$, the valuation of $i$ on bundle $S$ is denoted by $v_{i}(S) \geq 0$, assumed to be a non-negative integer. We impose the following restrictions on valuations of any buyer:

A1 Private Valuations: Each buyer knows his own valuation and it does not depend on the valuations of other buyers.

A2 Quasi-linear Utility: The utility or payoff of any buyer $i \in B$ on a bundle $S$ is given by $v_{i}(S)-p$, where $p$ is the price paid by buyer $i$ on bundle $S$. Also, if a buyer gets nothing and pays nothing, then his utility is zero: $v_{i}(\emptyset):=0 \forall i \in B$.

A3 Free Disposal (Monotonicity): $v_{i}(S) \leq v_{i}(T) \forall i \in B, \forall S, T \in \Omega$ with $S \subseteq T$.

A4 Zero Seller Valuations: The seller values the items at zero. His utility or payoff or revenue is the total payment he receives at a price. 
Assumptions A1-A4 are standard in literature. Unless stated explicitly, we do not pose any restriction on the valuations of the buyers besides these four assumptions and call them the general valuations.

Let $B_{-i}=B \backslash\{i\}$ be the set of buyers without buyer $i$. Let $\mathbb{B}=\left\{B, B_{-1}, \ldots, B_{-m}\right\}$. We will denote the economy with buyers only from set $M \subseteq B$ as $E(M)$. Whenever, $M \neq B$ and $M \in \mathbb{B}$, we call economy $E(M)$ a marginal economy. $E(B)$ is called the main economy.

We now define the combinatorial allocation problem [30]. The combinatorial allocation problem seeks to find an efficient allocation of the main economy. Let $X$ denote a feasible allocation in economy $E(M)(M \in \mathbb{B})$. Allocation $X$ is a vector of bundles on buyers in $M$ such that $X_{i} \cap X_{j}=\emptyset$ for any $i \neq j$. Allocation $X$ assigns bundle $X_{i}$ to buyer $i$ for every $i \in M$. The possibility of $X_{i}=\emptyset$ is allowed. We will denote the set of all feasible allocations of economy $E(M)$ as $\mathbb{X}(M)$.

An allocation $X$ is efficient in economy $E(M)$ if there does not exist another allocation $Y \in \mathbb{X}(M)$ such that $\sum_{i \in M} v_{i}\left(Y_{i}\right)>\sum_{i \in M} v_{i}\left(X_{i}\right)$. From assumption A3, every efficient allocation $X \in \mathbb{X}(M)$ should have $\bigcup_{i \in M} X_{i}=A$.

The problem of finding an efficient allocation can be formulated as a linear program [7]. Let $y_{i}(S) \in\{0,1\}$ be a variable which is assigned value 1 if a buyer $i \in B$ is allocated a bundle $S \in \Omega(S=\emptyset$ is allowed) and assigned zero otherwise. Let $z(X) \in\{0,1\}$ be a variable which is assigned 1 if allocation $X \in \mathbb{X}(M)$ is selected. The efficient allocation problem of economy $E(M)$ (for any $M \subseteq B$ ) is as follows:

$$
V(M)=\max _{y, z} \sum_{i \in M} \sum_{S \in \Omega} v_{i}(S) y_{i}(S)
$$

s.t.

$$
\begin{aligned}
\sum_{S \in \Omega} y_{i}(S) & =1 \quad \forall i \in M . \\
\sum_{X \in \mathbb{X}(M)} z(X) & =1 . \\
y_{i}(S) & =\sum_{X: X_{i}=S} z(X) \quad \forall S \in \Omega, \forall i \in M . \\
y_{i}(S) & \geq 0 \quad \forall i \in M, \forall S \in \Omega . \\
z(X) & \geq 0 \quad \forall X \in \mathbb{X}(M) .
\end{aligned}
$$

Given this, the dual problem is defined as:

$$
V(M)=\min _{\pi, \pi^{s}, p} \pi^{s}+\sum_{i \in M} \pi_{i}
$$

s.t.

$$
\begin{aligned}
& \pi^{s} \geq \sum_{i \in M} p_{i}\left(X_{i}\right) \quad \forall X \in \mathbb{X}(M) . \\
& \pi_{i} \geq v_{i}(S)-p_{i}(S) \quad \forall i \in M, \forall S \in \Omega .
\end{aligned}
$$


Dual variables $p_{i}(S)$ can be interpreted as the price on bundle $S$ to buyer $i$, with $\pi_{i}$ being the maximum payoff to buyer $i$ across all bundles and $\pi^{s}$ being the maximum payoff to the seller across all allocations in $\mathbb{X}(M)$.

Define the demand set of buyer $i$ at price vector $p \in \mathbb{R}_{+}^{|M| \times|\Omega|}$ as

$$
D_{i}(p):=\left\{S \in \Omega: v_{i}(S)-p_{i}(S) \geq v_{i}(T)-p_{i}(T) \forall T \in \Omega\right\}
$$

and the supply set of the seller at price vector $p \in \mathbb{R}_{+}^{|M| \times|\Omega|}$ in economy $E(M)$ as

$$
L(p):=\left\{X \in \mathbb{X}(M): \sum_{i \in M} p_{i}\left(X_{i}\right) \geq \sum_{i \in M} p_{i}\left(Y_{i}\right) \forall Y \in \mathbb{X}(M)\right\} .
$$

Definition 1 (Competitive Equilibrium) Price vector $p \in \mathbb{R}_{+}^{|M| \times|\Omega|}$ (feasible solution of $(\boldsymbol{D P}(M))$ ) and allocation $X$ (feasible solution of $(\boldsymbol{P}(M))$ ) are a competitive equilibrium (CE) of economy $E(M)$ for some $M \subseteq B$ if $X \in L(p)$, and $X_{i} \in D_{i}(p)$ for every buyer $i \in M$. Price $p$ is called a $C E$ price vector of economy $E(M)$.

From standard duality theory we can understand why the allocation supported in CE prices is efficient. ${ }^{2}$ Given feasible solution $(y, z)$ to $(\mathbf{P}(M))$ and feasible solution $\left(p, \pi, \pi^{s}\right)$ to $(\mathbf{D P}(M))$, we have the following complementary slackness (CS) conditions:

$$
\begin{aligned}
y_{i}(S)\left[\pi_{i}-\left[v_{i}(S)-p_{i}(S)\right]\right] & =0 & & \forall i \in M, \forall S \in \Omega . \\
z(X)\left[\pi^{s}-\sum_{i \in M} p_{i}\left(X_{i}\right)\right] & =0 & & \forall X \in \mathbb{X}(M) .
\end{aligned}
$$

Solutions $(y, z)$ and $\left(\pi, \pi^{s}, p\right)$ are optimal for the primal and dual problems respectively if and only if these CS conditions hold. From this we recover the standard intuition for CE prices: if $y_{i}(S)=1$ then $S \in D_{i}(p)$ from CS-1 (buyers receive a bundle in their demand set) and if $z(X)=1$ then $X \in L(p)$ from CS-2 (the seller maximizes his revenue).

In the rest of the paper, every price vector $p$ will be defined on $p \in \mathbb{R}_{+}^{|B| \times|\Omega|}$ (unless stated otherwise) and the projection of $p$ on $\mathbb{R}_{+}^{|M| \times|\Omega|}$ will be denoted as $p_{M}$ (or, $p_{-i}$ if $M=B_{-i}$ ). A component of $p_{M}$ will still be denoted as $p_{i}(\cdot)$ for every $i \in M$. For simplicity, we will often denote $\pi_{i}(p)$ as the payoff of buyer $i$ and $\pi^{s}\left(p_{M}\right)$ as the payoff or revenue of the seller in economy $E(M)$ at prices $p$. If we are considering buyers from set $M$ only, then the vector of payoffs of buyers from $M$ is simply denoted as $\pi_{M}(p)$ (or, simply $\pi_{-i}(p)$ if $M=B_{-i}$ ).

Definition 2 (Universal Competitive Equilibrium Price) A price vector $p$ is a universal competitive equilibrium (UCE) price vector if $p_{M}$ is a CE price vector of economy $E(M)$ for every $M \in \mathbb{B}$.

We provide some examples to illustrate the idea of UCE prices in Section 2.3. For now, we note that UCE prices do not require any similarity between the allocations that are supported in the $\mathrm{CE}$ of each of the marginal economies. Also, UCE prices always exist since $p:=v$ are (trivial) $\mathrm{UCE}$ prices.

\footnotetext{
${ }^{2}$ Bikhchandani and Ostroy [7] were the first to observe that non-anonymous and non-linear CE prices support the efficient allocation in the combinatorial allocation problem.
} 


\subsection{Vickrey Payments and UCE Prices}

The Vickrey-Clarke-Groves (VCG) mechanism $[31,8,15]$ is an ex post efficient and ex post individually rational direct revelation mechanism for which truth revelation is a dominant strategy (i.e., it is strategyproof, or truthful). Given submitted valuation profiles $\hat{v}=\left(\hat{v}_{1}, \ldots, \hat{v}_{m}\right)$, $\hat{v}_{i}$ representing the submitted valuation function (a vector on bundles) of buyer $i$, the VCG mechanism solves the efficient allocation problem for the main economy and the marginal economies. The implemented allocation $X^{*}$ is an efficient allocation in the main economy and the payment for buyer $i$ is calculated as $p_{i}^{v c g}=\hat{v}_{i}\left(X_{i}^{*}\right)-\left[V(B)-V\left(B_{-i}\right)\right]$. We refer to a buyer's (equilibrium) payoff in the VCG mechanism, which is his marginal product $V(B)-V\left(B_{-i}\right)$ as the Vickrey payoff and the payment as the Vickrey payment.

Consider the problem of determining Vickrey payments from a $\mathrm{CE}(p, X)$. We show that it is necessary and sufficient that $p$ is a UCE price vector.

Theorem 1 Let $(p, X)$ be a CE of the main economy. The Vickrey payments of every buyer can be calculated from $(p, X)$ if and only if $p$ is a UCE price vector. Moreover, if $p$ is a UCE price vector, then for every buyer $i \in B$, the Vickrey payment of buyer $i$ is $p_{i}^{v c g}=p_{i}\left(X_{i}\right)-\left[\pi^{s}(p)-\pi^{s}\left(p_{-i}\right)\right]$.

Proof: Sufficiency of UCE Prices: Consider a buyer $i \in B$. Let $\left(p_{-i}, Y\right)$ be a CE of economy $E\left(B_{-i}\right)$. From the definition of Vickrey payment, we have:

$$
\begin{aligned}
p_{i}^{v c g} & =v_{i}\left(X_{i}\right)-\left[V(B)-V\left(B_{-i}\right)\right] . \\
& =v_{i}\left(X_{i}\right)-\sum_{j \in B}\left[\left[v_{j}\left(X_{j}\right)-p_{j}\left(X_{j}\right)\right]+p_{j}\left(X_{j}\right)\right]+\sum_{j \in B_{-i}}\left[\left[v_{j}\left(Y_{j}\right)-p_{j}\left(Y_{j}\right)\right]+p_{j}\left(Y_{j}\right)\right] \\
& =p_{i}\left(X_{i}\right)-\sum_{j \in B} p_{j}\left(X_{j}\right)+\sum_{j \in B_{-i}} p_{j}\left(Y_{j}\right) \\
& -\sum_{j \in B_{-i}}\left[v_{j}\left(X_{j}\right)-p_{j}\left(X_{j}\right)\right]+\sum_{j \in B_{-i}}\left[v_{j}\left(Y_{j}\right)-p_{j}\left(Y_{j}\right)\right] \text { (rearranging terms). }
\end{aligned}
$$

Since $(p, X)$ is a $\mathrm{CE}$ of the main economy we have $X_{j} \in D_{j}(p)$ for every $j \in B$. Similarly, $\left(p_{-i}, Y\right)$ is a $\mathrm{CE}$ of economy $E\left(B_{-i}\right)$. So, $Y_{j} \in D_{j}(p)$ for every $j \in B_{-i}$. This means $v_{j}\left(X_{j}\right)-p_{j}\left(X_{j}\right)=v_{j}\left(Y_{j}\right)-p_{j}\left(Y_{j}\right)$ for every $j \in B_{-i}$. This cancels terms in Equation 1 and transforms it as $p_{i}^{v c g}=p_{i}\left(X_{i}\right)-\left[\sum_{j \in B} p_{j}\left(X_{j}\right)-\sum_{j \in B_{-i}} p_{j}\left(Y_{j}\right)\right]=p_{i}\left(X_{i}\right)-\left[\pi^{s}(p)-\pi^{s}\left(p_{-i}\right)\right]$.

Necessity of UCE Prices: Construct the valuation profile $v^{\prime}$ as $v_{i}^{\prime}(S):=p_{i}(S)$ for every $i \in B$ and every $S \in \Omega . p$ is a CE price vector of the main economy at valuation profile $v^{\prime}$. The Vickrey payment of every buyer $i \in B$ at valuation profile $v^{\prime}$ is $v_{i}^{\prime}\left(X_{i}\right)-V(B)+V\left(B_{-i}\right)=$ $p_{i}\left(X_{i}\right)-\pi^{s}(p)+\pi^{s}\left(p_{-i}\right)$. Since the Vickrey payments are calculated only from $(p, X)$, they should be calculated in the same manner for all the valuation valuation profiles for which $(p, X)$ is a $\mathrm{CE}$ of the main economy. This means, for every buyer $i \in B$, the Vickrey payment should be calculated as: $p_{i}\left(X_{i}\right)-\pi^{s}(p)+\pi^{s}\left(p_{-i}\right)$. 
Assume for contradiction that $p$ is a $\mathrm{CE}$ price vector for the main economy but not a UCE price vector. This means, for some marginal economy $E\left(B_{-j}\right), p_{-j}$ is not a CE price vector. $\left(p_{-j}, \pi_{-j}(p), \pi^{s}\left(p_{-j}\right)\right)$ constitute a dual feasible solution of formulation $\left(\mathbf{D P}\left(B_{-j}\right)\right)$ but not an optimal solution since $p_{-j}$ is not a CE price vector of $E\left(B_{-j}\right)$. So, we can write $\sum_{i \in B_{-j}} \pi_{i}(p)+\pi^{s}\left(p_{-j}\right)<V\left(B_{-j}\right)$. Since $(p, X)$ is a CE of the main economy, we have $\sum_{i \in B} \pi_{i}(p)+\pi^{s}(p)=V(B)$. Substituting into the standard expression for the Vickrey payment of buyer $j$, we have:

$$
p_{j}^{v c g}=v_{j}\left(X_{j}\right)-V(B)+V\left(B_{-j}\right)<p_{j}\left(X_{j}\right)-\pi^{s}(p)+\pi^{s}\left(p_{-j}\right) .
$$

This gives us a contradiction.

We refer to the term $\pi^{s}(p)-\pi^{s}\left(p_{-i}\right)$ as the discount for buyer $i$ and the term $p_{i}\left(X_{i}\right)-$ $\left[\pi^{s}(p)-\pi^{s}\left(p_{-i}\right)\right]$ as the discounted price for buyer $i$. Notice that the claims in Theorem 1 continue to hold for restricted classes of valuations and for simpler prices such as anonymous or item prices. Also, notice that the adjustment reduces immediately to the standard VCG payment definition for UCE prices $p:=v$.

Before continuing to provide some examples of this correspondence between UCE prices and Vickrey payments we define the following restricted class of valuations:

Definition 3 (Buyers are Substitutes) We say buyers are substitutes $(B A S)$ if $V(B)$ $V(K) \geq \sum_{i \in B \backslash K}\left[V(B)-V\left(B_{-i}\right)\right] \forall K \subseteq B$.

Intuitively, BAS holds when buyers are more alike than different and contribute decreasing marginal product as the size of the economy grows. The gross substitutes condition, familiar in economics, implies that BAS holds [5]. BAS is important in the current context because it exactly characterizes the restriction on valuations required for each buyer to simultaneously receive his Vickrey payoff at some CE price vector [7].

\subsection{Examples}

We now give some examples to illustrate the concept of UCE prices and the discounted prices which give Vickrey payments. Table 1 (a) illustrates a problem with two buyers and two items and valuations. It is easy to verify that buyers are substitutes in this example. A UCE price vector $(p)$ in this example is $p_{1}(\emptyset)=p_{2}(\emptyset)=0, p_{1}(\{1\})=p_{1}^{v c g}=6, p_{1}(\{2\})=8$, $p_{1}(\{1,2\})=p_{2}(\{1,2\})=10, p_{2}(\{1\})=2, p_{2}(\{2\})=p_{2}^{v c g}=4$. In the main economy, the efficient allocation (buyer 1 gets item 1 and buyer 2 gets item 2) is supported at $p$. In the marginal economy with only buyer 1, efficient allocation (buyer 1 gets both items) is supported at $p_{-2}$. Also, in the marginal economy with only buyer 2 , efficient allocation (buyer 2 gets both items) is supported at $p_{-1}$. Also, observe that Vickrey payments are directly calculated (without discounts) at this UCE price vector.

Table 1 (b) provides an example with three buyers and two items. It is easy to verify that buyers are not substitutes in this example. A UCE price vector $(p)$ is the following: 


\begin{tabular}{|c|cccc|}
\hline & $\emptyset$ & $\{1\}$ & $\{2\}$ & $\{1,2\}$ \\
\hline \hline 1 & 0 & 8 & 9 & 12 \\
\hline 2 & 0 & 6 & 8 & 14 \\
\hline
\end{tabular}

(a) Buyers are substitutes.

\begin{tabular}{|c|cccc|}
\hline & $\emptyset$ & $\{1\}$ & $\{2\}$ & $\{1,2\}$ \\
\hline \hline 1 & 0 & 3 & 0 & 3 \\
\hline 2 & 0 & 0 & 6 & 6 \\
\hline 3 & 0 & 0 & 2 & 4 \\
\hline
\end{tabular}

(b) Buyers are not substitutes.

Table 1: Examples to show the correspondence between UCE prices and Vickrey payments

$p_{1}(\emptyset)=p_{2}(\emptyset)=p_{3}(\emptyset)=0, p_{1}(\{1\})=2, p_{1}(\{2\})=0, p_{1}(\{1,2\})=2, p_{2}(\{1\})=0, p_{2}(\{2\})=$ $4, p_{2}(\{1,2\})=4, p_{3}(\{1\})=0, p_{3}(\{2\})=2, p_{3}(\{1,2\})=4$. In the main economy and in the marginal economy with buyers 1 and 2 only, the efficient allocation (buyer 1 gets item 1 , buyer 2 gets item 2 and buyer 3 gets nothing) is supported at $p_{-3}$. In the marginal economy with buyers 1 and 3 only, the efficient allocation (buyer 1 gets item 1 and buyer 3 gets item 2 ) is also supported at $p_{-2}$. Similarly, in the marginal economy with buyers 2 and 3 only, the efficient allocation (buyer 2 gets item 2 and buyer 3 gets item 1 ) is supported at $p_{-1}$. The Vickrey payments for buyers can be calculated as: $p_{1}^{v c g}=p_{1}(\{1\})-\left[\pi^{s}(p)-\pi^{s}\left(p_{-1}\right)\right]=$ $2-[6-4]=0, p_{2}^{v c g}=p_{2}(\{2\})-\left[\pi^{s}(p)-\pi^{s}\left(p_{-2}\right)\right]=4-[6-4]=2, p_{3}^{v c g}=0$.

\section{A Class of Ascending Price Vickrey Auctions}

We provide a relaxation of the traditional definition of ascending price auctions to retain a single price path but allow for final payments to be determined as an adjustment from (final) clearing prices. We introduce a "black-box model" for the general class of ascending price Vickrey auctions that fall within this definition, and provide ex post efficiency and equilibrium claims for any auction in this class. This reverses a negative result in de Vries et al. [11], which holds for a more restricted auction definition. In addition, we demonstrate that auctions within our class must maintain both non-anonymous and non-linear prices to achieve the VCG outcome, even in the special cases of gross substitutes valuations.

The specific auction protocols, namely a generalization of the primal-dual auction in de Vries et al. [11] and the subgradient auction in Parkes and Ungar [27], are defined in subsequent sections. In Sections 3.1 to 3.3 we will assume that buyers submit true demand sets in each round, i.e. bid truthfully. This bidding strategy is shown to be an ex post Nash equilibrium in Section 3.4.

\subsection{A Relaxed Definition of Ascending Price Auctions}

In defining a class of ascending price auctions we mainly follow de Vries et al. [11] and Gul and Stacchetti [16]. The main difference is that we relax the requirement that the final prices in the auction define payments of buyers. First, we define the notion of a price path: 
Definition 4 A price path is any of these four types of functions:

- Linear and anonymous price path: $P: \mathbb{T} \rightarrow \mathbb{R}_{+}^{|A|}$,

- Linear and non-anonymous price path: $P: \mathbb{T} \rightarrow \mathbb{R}_{+}^{|A| \times|B|}$,

- Non-linear and anonymous price path: $P: \mathbb{T} \rightarrow \mathbb{R}_{+}^{|\Omega|}$,

- Non-linear and non-anonymous price path: $P: \mathbb{T} \rightarrow \mathbb{R}_{+}^{|\Omega| \times|B|}$,

where $\mathbb{T}$ denotes the set of rounds in an auction and $t \in \mathbb{T}$ denotes a round in an auction with $P(t)$ denoting a price vector seen at time $t$. A price path is ascending if $P(t)$ is nondecreasing with time.

Definition 5 (Ascending Price Auction) An ascending price auction is a single ascending price path $P(\cdot)$ which starts from $P(0)$ and ends at $P(T)$ with an allocation and payment for buyers such that:

$C 1$ At every round $t$, buyers report their demand set (bids) at price vector $P(t)$.

C2 For every round $t \in \mathbb{T}$, the price adjustment is determined only by current price vector $P(t)$ and current demand set information: $D_{i}(P(t))$ for every buyer $i \in B$.

C3 Every buyer $i \in B$ gets a bundle (possibly $\emptyset)$ from his demand set $D_{i}(P(T))$ at the end of the auction.

$C_{4}$ The final payment of buyers is determined from the final allocation and price vector in the last round $(P(T))$ only.

Many auctions in the literature fall into this class of ascending price auctions including the single-path auctions in Ausubel [2] and de Vries et al. [11]. ${ }^{3} 4$

One notable exception is Ausubel's multi-item auction [1], which maintains multiple price paths, each linear and anonymous, and performs clinching and unclinching of items in determining payments dynamically during the auction. This auction achieves the VCG outcome for gross substitutes valuations. Similarly, the multi-path variation of the auction in de Vries et al. [11] lies outside of this class.

The traditional definition of ascending price auctions, as formalized by de Vries et al. [11] is the following. We call it ascending price(0) auctions to indicate that no adjustment to final prices is done in these auctions to calculate payments of buyers.

\footnotetext{
${ }^{3}$ The auction of Ausubel and Milgrom [3] can also be provided with a non-proxied, ascending-price interpretation, at which point it is equivalent to the $i$ Bundle auction [24].

${ }^{4}$ Ausubel's auction [2] for homogeneous items appears not to fall within this class because it performs clinching of items during the auction with linear and anonymous prices. However, it can be reinterpreted as an auction that maintains a single non-linear and non-anonymous price path and terminates with a $\mathrm{CE}$ price vector that gives the Vickrey payments for non-increasing marginal valuations [6].
} 
Definition 6 (Ascending Price(0) Auctions) An ascending price(0) auction is an ascending price auction with Step C4 modified as follows:

C4' The final payment of buyers is the final price seen by them on their respective final allocation.

Clearly, every ascending price(0) auction is also an ascending price auction. Besides the multi-path auctions discussed, all previously known auctions are ascending price(0) auctions. For such auctions, de Vries et al. provide the following negative result.

Theorem 2 (de Vries et al. [11]) Suppose there are two items and at least three buyers. If the valuation of one of the buyers fail the gross substitutes condition, then there exists a class of valuations for other buyers satisfying the gross substitutes condition such that no ascending price(0) auction can terminate with VCG prices for these valuations when buyers bid truthfully.

The proof of this Theorem is done by constructing a parametric valuation profile with valuations of all but one buyer satisfying the gross substitutes condition and showing that the VCG payments of buyers depend on the parameter but the final prices of ascending price $(0)$ auctions do not. To overcome this negative result of Theorem 2, we propose ascending price auctions by relaxing condition [C4'] in Definition 6 to [C4].

We consider ascending price auctions in which truthful submission of demand sets in response to prices in each round is an ex post Nash equilibrium in the sense of Jehiel et al. [17].

Definition 7 Truthful bidding in every round of an auction is an ex post Nash equilibrium if for every buyer $i \in B$, if buyers in $B_{-i}$ follow the truthful bidding strategy, then buyer $i$ maximizes his payoff in the auction by following the truthful bidding strategy.

A strategy profile that is an ex post Nash equilibrium is invariant to the private valuation of each buyer. This makes it appropriate for auctions in which buyers have incomplete information about valuations of others. ${ }^{5} 6$

As we will elaborate later, prices in our auctions act as means for eliciting preferences and not as prices paid by buyers in the end. Our auctions will involve discounts for buyers at the end (or, discounts calculated incrementally across rounds). Truthful bidding does not take into account such discounts while calculating bids (demand sets). Truthful bidding involves, for every buyer, calculation of demand sets with respect to true valuation and current auction prices (without any discounts).

\footnotetext{
${ }^{5}$ An ex post Nash equilibrium is also a Bayesian Nash equilibrium because a buyer maximizes his payoff in an ex post Nash equilibrium for any belief about the valuations of other buyers, but more robust [17].

${ }^{6}$ Ex post Nash equilibrium has been adopted as a solution concept in other ascending price auction models, for instance in [11]. Ex post implementation has also been adopted for direct-revelation mechanisms with interdependent values, see for instance [21] .
} 
More specifically, we are concerned with efficient ascending price auctions, i.e., auctions in which truthful bidding is an ex post Nash equilibrium strategy and in which the auction terminates with an efficient allocation.

Proposition 1 If $X$ is the final allocation and $p$ is the final price vector in an efficient ascending price auction, then $(p, X)$ is a $C E$ of the main economy. ${ }^{7}$

Proof: First, $X$ is an efficient allocation of the main economy by the definition of efficient ascending price auction. Any ascending price auction selects a final allocation from the price and demand set information in the final round. Thus, if $X$ is an efficient allocation, then it is an efficient allocation for all valuations consistent with demand set profile $D(p):=$ $\left(D_{1}(p), \ldots, D_{n}(p)\right)$.

By property (C3) of an ascending price auction, $X_{i} \in D_{i}(p)$ for all $i \in B$. Assume for contradiction $X \notin L(p)$. Now, consider the valuation profile $v^{\epsilon}$ as follows. If $\emptyset \notin D_{i}(p)$, then

$$
v_{i}^{\epsilon}(S)= \begin{cases}p_{i}(S)+\epsilon & \forall S \in D_{i}(p) \\ p_{i}(S) & \text { otherwise }\end{cases}
$$

for some small $\epsilon>0$. If $\emptyset \in D_{i}(p)$, then

$$
v_{i}^{\epsilon}(S)= \begin{cases}p_{i}(S) & \forall S \in D_{i}(p) \\ p_{i}(S)-\epsilon & \text { otherwise }\end{cases}
$$

Clearly, $v^{\epsilon}$ is consistent with $D(p)$. Consider an allocation $\hat{X} \in L(p)$. There can be a maximum of $\min (m, n)$ non-empty bundles in $\hat{X}$. Since $\hat{X} \in L(p)$, we can find small enough $\epsilon>0\left(\epsilon<\frac{\sum_{i \in B}\left[p_{i}\left(\hat{X}_{i}\right)-p_{i}\left(X_{i}\right)\right]}{\min (m, n)}\right)$ such that $\sum_{i \in B} v_{i}^{\epsilon}\left(\hat{X}_{i}\right)>\sum_{i \in B} v_{i}^{\epsilon}\left(X_{i}\right)$. This means, $X$ is not an efficient allocation for valuation profile $v^{\epsilon}$ for some small enough $\epsilon>0$. This gives us a contradiction. So, $X \in L(p)$ and $X_{i} \in D_{i}(p)$ for every $i \in B$. Thus, $(p, X)$ is a CE of the main economy.

Using Proposition 1, we prove a stronger result for efficient ascending price auctions.

Proposition 2 An efficient ascending price auction in which all buyers that are allocated no items have zero payment must terminate with a UCE price vector.

Proof: From Proposition 1, an efficient ascending price auction must terminate at CE price vector of the main economy. From the revelation principle, the direct revelation mechanism of an efficient ascending price auction has an equilibrium in which truthful bidding is a dominant strategy. But Groves mechanisms are the only strategy-proof and efficient direct

\footnotetext{
${ }^{7}$ Results similar to Proposition 1 can be found in Nisan and Segal [22] and Parkes [25]. Nisan and Segal's result, applicable for a more general setting than combinatorial auctions, states that if we can determine an efficient allocation from a set of "messages" (demand sets and prices in our case), then we can also construct a CE price from these messages. These earlier results do not appear to imply our result because they do not require that the prices in the message are already $\mathrm{CE}$ prices.
} 
mechanisms [14]. Moreover, the VCG mechanism is the only Groves mechanism in which every loser pays nothing. This means, the final payment in an efficient ascending price auction in which every loser pays nothing is the Vickrey payment. From property (C4) of ascending price auctions, the final payments are determined from the final CE price vector and efficient allocation. From Theorem 1, this price vector must be a UCE price vector.

\subsection{Insufficiency of Simpler Prices}

Many known combinatorial auctions [24, 11, e.g.] maintain non-linear and non-anonymous prices. This requires maintaining an exponential number of prices in the auction in the worst case, although in practice one only needs to report explicit prices on bundles that receive bids.

We briefly consider whether one can maintain simpler prices and still achieve the VCG outcome in the auctions within our class. Since the definition of ascending price auctions requires termination with CE prices (Proposition 1) we have the following proposition.

Proposition 3 ([7]) Every efficient ascending price auction must allow a non-linear and non-anonymous price path.

We are interested to understand whether simpler price paths are sufficient for special cases, such as that of gross substitutes preferences. This is a broad class of preferences for which a linear and anonymous CE price vector exists [18], and thus interesting to consider.

Definition 8 (Gross Substitutes) A valuation function $v_{i}$ satisfies gross substitutes $(G S)$ if, for all price vectors $p, p^{\prime} \in \mathbb{R}_{+}^{|\Omega| \times|B|}$ such that $p_{i}(S)=\sum_{j \in S} p_{i}(\{j\}) \leq p_{i}^{\prime}(S)=$ $\sum_{j \in S} p_{i}^{\prime}(\{j\}), \forall i \in B, \forall S \in \Omega$ and for all $S \in D_{i}(p)$, there exists $S^{\prime} \in D_{i}\left(p^{\prime}\right)$ such that $\left\{j \in S: p_{i}(\{j\})=p_{i}^{\prime}(\{j\})\right\} \subseteq S^{\prime}$.

Informally, valuations satisfy gross substitutes (or simply substitutes) if a buyer continues to demand the same item when the price on another item increases.

Gul and Stacchetti [16] already established that a traditional ascending price auction with a linear and anonymous price path cannot terminate with VCG prices for substitutes valuations. On the other hand, Ausubel [1] showed that multiple anonymous and linear price paths are sufficient by cleverly using information collected from buyers in these price paths.

Considering anonymous but possibily non-linear prices, we can first observe that anonymous UCE prices only exist when BAS. To see this, notice that the discount is zero when prices are anonymous (by the definition of the price adjustment in Theorem 1), and thus prices must already support VCG payments if they are UCE. Yet, this equivalence between UCE and VCG payments (and thus between CE and VCG) requires BAS. So, non-anonymous prices will be required in most interesting cases.

We also have the following negative result, which shows that both non-linear and nonanonymous prices are required even for the restricted case of substitutes valuations. 
Proposition 4 An efficient ascending price auction in which losers pay nothing must maintain a non-linear and non-anonymous price path for substitutes valuations.

Proof: We will show that for the example in Table 1 (a), the the UCE price vectors are non-linear and non-anonymous. From Proposition 2, the result then follows.

Assume for contradiction that $p \in \mathbb{R}_{+}^{|B| \times|A|}$ is a UCE price vector for the example. It is easy to see that in the $\mathrm{CE}$ of $E(B)$, buyer 1 is assigned item 1 and in the $\mathrm{CE}$ of $E\left(B_{-2}\right)$, buyer 1 is assigned bundle $\{1,2\}$. This means, $8-p_{1}(\{1\})=12-\left[p_{1}(\{1\})+p_{1}(\{2\})\right]$. This gives us $p_{1}(\{2\})=4$. Similarly, buyer 2 is assigned item 2 in CE of $E(B)$ and bundle $\{1,2\}$ in $\mathrm{CE}$ of $E\left(B_{-1}\right)$. This means, $8-p_{2}(\{2\})=14-\left[p_{2}(\{1\})+p_{2}(\{2\})\right]$. This gives us, $p_{2}(\{1\})=6$. Now, since buyer 1 is assigned item 1 in CE of $E(B)$, we have $8-p_{1}(\{1\}) \geq$ $9-p_{1}(\{2\})=5$. This gives us, $p_{1}(\{1\}) \leq 3$. Also, the seller should maximize his utility in the $\mathrm{CE}$ allocation of $E(B)$. This means, $p_{1}(\{1\})+p_{2}(\{2\}) \geq p_{2}(\{1\})+p_{2}(\{2\})$. This gives us, $p_{1}(\{1\}) \geq p_{2}(\{1\})=6$. Putting these together, we have a contradiction. A similar argument shows that there does not exist $p \in \mathbb{R}_{+}^{|\Omega|}$, which is a UCE price vector in example in Table 1. A non-linear and non-anonymous UCE price vector is the "value=price" UCE price vector.

There are examples of auctions with anonymous and linear price paths, but only for very restricted valuations. For instance, in the unit-demand case there exists anonymous linear CE prices which give every buyer his Vickrey payoff [19], and Demange et al. [13] have designed an auction which ends with such CE prices.

\subsection{A General Class of Ascending Price Vickrey Auctions}

Continuing, we now define a black-box model for the general class of ascending price auctions to which our main results apply. The auctions maintain non-anonymous and non-linear prices and adjust prices until a UCE price vector is established.

As before, let $\pi_{i}(p):=\max _{S \in \Omega}\left[v_{i}(S)-p_{i}(S)\right]$ denote the maximum payoff of a buyer $i$ at price vector $p$, with $D_{i}(p)$ denoting his demand set. Also, let $\pi^{s}\left(p_{M}\right):=\max _{X \in \mathbb{X}(M)} \sum_{i \in M} p_{i}\left(X_{i}\right)$ denote the maximum revenue to the seller in economy $E(M)$ for every $M \in \mathbb{B}$ and $L\left(p_{M}\right)$ denoting the supply set of the seller. Notation $M^{+}(p):=\left\{i \in M: \emptyset \notin D_{i}(p)\right\}$ denotes buyers in economy $E(M)$ who do not have $\emptyset$ in their demand set. We define

$$
L^{*}\left(p_{M}\right):=\left\{X \in L\left(p_{M}\right): X_{i} \in D_{i}(p) \cup\{\emptyset\} \forall i \in M\right\} \subseteq L\left(p_{M}\right) \quad \forall M \subseteq B
$$

to denote the subset of the revenue maximizing allocations (if any) of the seller that assigns to every buyer either a bundle from his demand set or the $\emptyset$ bundle.

Definition 9 (Quasi-CE Price) Price vector $p \in \mathbb{R}_{+}^{|M| \times|\Omega|}$ is a quasi-CE (QCE) price vector of economy $E(M)$ for some $M \in \mathbb{B}$, if $L^{*}(p)$ is non-empty. Price vector $p \in \mathbb{R}_{+}^{|B| \times|\Omega|}$ is a universal $\boldsymbol{Q C E}(u Q C E)$ price vector if $p_{M}$ is a QCE price vector of economy $E(M)$ for every $M \in \mathbb{B}$. 
Intuitively, prices are QCE if demand is no less than supply and prices are universal QCE if demand is no less than supply in any marginal economy.

Using the notion of a $\mathrm{uQCE}$ price vector, we define a class of $u Q C E$-invariant auctions and show that any auction in this class maintains a $\mathrm{uQCE}$ price vector.

Definition 10 (uQCE-invariant Auctions) A uQCE-invariant auction is defined as follows:

SO The auction starts at the zero price vector.

S1 In round $t$ of the auction, with price vector $p^{t}$ :

S1.1 Collect demand sets of buyers at price vector $p^{t}$.

S1.2 If $p^{t}$ is a UCE price vector with respect to reported demand sets, then go to Step $\mathrm{S} 2$.

S1.3 Else, select a set of adjusted buyers $\emptyset \neq U^{t} \subseteq B^{+}\left(p^{t}\right)^{8}$ who will see a price increase.

S1.4 If $i \in U^{t}$ and $S \in D_{i}\left(p^{t}\right)$, then $p_{i}^{t+1}(S):=p_{i}^{t}(S)+1$. Else, $p_{i}^{t+1}(S):=p_{i}^{t}(S)$. Repeat from Step S1.1.

S2 The auction ends with final allocation of the auction being any $X \in L^{*}\left(p^{T}\right)$ and final payment of every buyer $i \in B$ being $p_{i}^{T}\left(X_{i}\right)-\left[\pi^{s}\left(p^{T}\right)-\pi^{s}\left(p_{-i}^{T}\right)\right]$, where $p^{T}$ is the final price vector of the auction.

The uQCE-invariant auctions clearly fall within the class of auctions introduced in Definition 5. A single price vector is maintained in each round, and buyers respond with demand sets. Notice that prices are only increased to buyers, $U^{t}$, that still report a non-empty demand set (Step S1.3), and that prices on all bundles in such a buyer's current demand set are increased by unity (Step S1.4). The price adjustment suggested in Theorem 1 is adopted on termination (Step S2).

The price adjustment process (selection of $U^{t}$ in Step $S 1.3$ ) defines a class of uQCEinvariant auctions. Besides $\emptyset \neq U^{t} \subseteq B^{+}\left(p^{t}\right)$, we place no restriction on the choice of $U^{t}$. For various selections of adjusted buyers, we will get different auctions. All will be valid ascending price Vickrey auctions. However, the specifics will affect both the simplicity and transparency of the auction as well as the speed of termination (see Section 3.5).

The auctions in this class may be called clock auctions because they maintain an ask price and require that buyers state demand sets in each round. Buyers do not submit bid prices. The traditional role of winner determination is still present, even if implicitly, and even though feedback to buyers can be limited to ask prices and need not include information about a provisional allocation. Winner determination is a natural way to test for UCE prices

${ }^{8} B^{+}\left(p^{t}\right)$, like $M^{+}\left(p^{t}\right)$, is defined as the set of all buyers in $B$ who do not demand the $\emptyset$ bundle. 
and thus termination (Step S1.2) and also to determine the set of adjusted buyers that will be used to define price updates (Step S1.3).

Here are two simple restrictions on the uQCE-invariant auctions:

- A $\boldsymbol{u} \boldsymbol{Q C E}$-invariant(0) auction is a uQCE-invariant auction with Step S2 modified as follows:

S2(0) The auction ends with the final allocation of the auction being any $X \in L^{*}(p)$ and final payment of every buyer $i \in B$ being $p_{i}\left(X_{i}\right)$, where $p$ is the final price vector of the auction.

- A $u Q C E$-invariant auction for the main economy is a UQCE-invariant auction with Step S1.2 modified as:

S1.2m If $p^{t}$ is a CE price vector of the main economy, then go to Step S2.

- A $u Q C E$-invariant(0) auction for the main economy is a $\mathrm{UQCE}$-invariant auction for the main economy with Step S2 modified as Step S2(0).

Most auctions in the literature are $u Q C E$-invariant(0) auctions for the main economy. Examples include auctions in de Vries et al. [11], Ausubel and Milgrom [3], Parkes [24] and Bikhchandani and Ostroy [6]. These uQCE-invariant(0) auctions for the main economy can be easily converted to uQCE-invariant auctions for the main economy, and thus ascending price Vickrey auctions for general valuations. We extend two such auctions to uQCE-invariant auctions in Sections 4 and 5.

If a uQCE-invariant auction is not a uQCE-invariant(0) auction, then the prices in such an auction act as a means to elicit preferences of buyers. Payments of buyers are calculated as a function of auction prices but need not equal the auction prices. Importantly, buyers need to respond to auction prices, without considering discounts, for bidding. As we will show, doing so truthfully will constitute an ex post Nash equilibrium for buyers in every uQCE-invariant auction.

Our first result is a theorem showing that every uQCE-invariant auction maintains uQCE prices in every round and terminates with UCE prices.

Theorem 3 Every uQCE-invariant auction achieves the VCG outcome if all buyers submit their true demand sets in each round.

Proof: Let $p^{t}$ be the price vector in round $t$ of a uQCE-invariant auction.

Lemma 1 In any round $t$ of a $u Q C E$-invariant auction for every buyer $i \in B$ the demandset weakly increases with $D_{i}\left(p^{t}\right) \subseteq D_{i}\left(p^{t+1}\right)$.

\footnotetext{
${ }^{9}$ Although $i$ Bundle [24] also reports the provisional allocation in each round, this is not necessary for the functioning of the auction.
} 
Proof: From the price adjustment and the starting price vector, the price vector in a uQCEinvariant auction is an integer vector. In any round the auction, for every buyer $i \in U^{t}$, the prices of bundles demanded by $i$ are increased by unity. Since valuations of buyers are assumed to be integers, the change in payoff of $i$ from such a price adjustment is -1 . Since price of any buyer not in $U^{t}$ is unchanged, their payoff is also unchanged. This implies that demand set of every buyer weakly increases after a price adjustment, i.e., $D_{i}\left(p^{t}\right) \subseteq D_{i}\left(p^{t+1}\right)$ for any non-terminal round $t$ in the auction.

From Lemma 1, if $S \notin D_{i}\left(p^{t}\right)$, then $S$ was never demanded by $i$ in any round before $t$. From the starting price and price adjustment rule of the auction, this further implies that if $S \notin D_{i}\left(p^{t}\right)$, then $p_{i}^{t}(S)=0$. Now, consider economy $E(M)$ for any $M \in \mathbb{B}$. Clearly, $L\left(p_{M}^{t}\right)$ is non-empty. Consider $X \in L\left(p_{M}^{t}\right)$. Construct allocation $Y \in \mathbb{X}(M)$ as $Y_{i}=X_{i}$ if $X_{i} \in D_{i}\left(p^{t}\right)$ and $Y_{i}=\emptyset$ otherwise. As argued before, for any $i \in M$, if $X_{i} \notin D_{i}\left(p^{t}\right)$, then $p_{i}^{t}\left(X_{i}\right)=0=p_{i}^{t}(\emptyset)=p_{i}^{t}\left(Y_{i}\right)$. This means, $\sum_{i \in M} p_{i}^{t}\left(X_{i}\right)=\sum_{i \in M} p_{i}^{t}\left(Y_{i}\right)$. This further means that $Y \in L\left(p_{M}^{t}\right)$. By the definition of $Y, Y \in L^{*}\left(p_{M}^{t}\right)$ indicating non-emptiness of $L^{*}\left(p_{M}^{t}\right)$. This means, the price vector in every round of a $\mathrm{uQCE}$-invariant auction is a uQCE price vector.

For the second claim, observe that in any round $t$ of a uQCE-invariant auction $U^{t} \subseteq$ $B^{+}\left(p^{t}\right)$. This means, if $i \notin B^{+}\left(p^{t}\right)$, then the price of buyer $i$ will not increase in any round $t$ of a uQCE-invariant auction. Due to unit price increase, the valuations of a buyer provides an upper bound on the price in a uQCE-invariant auction. Since valuations of buyers are finite, every uQCE-invariant auction will terminate finitely. By the termination condition in Step $S 1.2$ of Definition 10, the final price vector is a UCE price vector and the final allocation is an efficient allocation of the main economy. From the payment rule in Step S2 and Theorem 1, every uQCE-invariant auction achieves a VCG outcome.

Using arguments similar to Lemma 1, it is also simple to show that the prices in every round of uQCE-invariant auctions for the main economy and uQCE-invariant(0) auctions for the main economy are $\mathrm{uQCE}$ prices. Given this, we can also consider the special case of uQCE-invariant auctions for the main economy.

Theorem 4 If buyers are substitutes, then every uQCE-invariant auction for the main economy achieves the VCG outcome if buyers submit true demand sets in each round.

Proof: On termination of a uQCE-invariant auction for the main economy we have a uQCE price vector that is also a $\mathrm{CE}$ price vector in the main economy. We show that such a price vector is a UCE price vector when BAS holds. The result follows from Theorem 1.

Let $p$ be a uQCE price vector. Consider a buyer $i \in B$. Since $p_{-i}$ is a QCE price vector in economy $E\left(B_{-i}\right)$, consider $X \in L^{*}\left(p_{-i}\right)$ and let $K=\left\{k \in B_{-i}: X_{k} \neq \emptyset\right\}$. Now,

$$
\pi^{s}\left(p_{-i}\right)=\sum_{k \in K} p_{k}\left(X_{k}\right)=\sum_{k \in K}\left[v_{k}\left(X_{k}\right)-\pi_{k}(p)\right] \leq V(K)-\sum_{k \in K} \pi_{k}(p) .
$$


Now,

$$
\begin{aligned}
& \pi_{i}(p)+\pi^{s}(p)-\pi^{s}\left(p_{-i}\right) \\
& \geq \pi_{i}(p)+\pi^{s}(p)-V(K)+\sum_{k \in K} \pi_{k}(p) \text { (From Equation 3) } \\
& =\pi_{i}(p)+V(B)-\sum_{k \in B} \pi_{k}(p)-V(K)+\sum_{k \in K} \pi_{k}(p) \text { (Since } p \text { is a CE) } \\
& =V(B)-V(K)-\sum_{k \in B \backslash(K \cup\{i\})} \pi_{k}(p) \\
& \geq \sum_{k \in B \backslash K} \pi_{k}^{v c g}-\sum_{k \in B \backslash(K \cup\{i\})} \pi_{k}(p) \text { (Since buyers are substitutes) } \\
& \geq \sum_{k \in B \backslash K} \pi_{k}^{v c g}-\sum_{k \in B \backslash(K \cup\{i\})} \pi_{k}^{v c g}=\pi_{i}^{v c g}
\end{aligned}
$$

The last inequality comes from a result in Bikhchandani and Ostroy [7] which states that under BAS the core payoff (payoff of buyers from a CE price vector) vectors form a lattice and the unique maximum core payoff is the Vickrey payoff vector. But, $\pi_{i}(p)+\pi^{s}(p)-$ $\pi^{s}\left(p_{-i}\right)=\sum_{k \in B} \pi_{k}(p)-\sum_{k \in B_{-i}} \pi_{k}(p)+\pi^{s}(p)-\pi^{s}\left(p_{-i}\right) \leq V(B)-V\left(B_{-i}\right)=\pi_{i}^{v c g}$, where the inequality comes from the fact that $\left(p_{-i}, \pi_{-i}(p), \pi^{s}\left(p_{-i}\right)\right)$ is a dual feasible solution of formulation $\left(\mathbf{D P}\left(B_{-i}\right)\right)$. This means, for BAS, that $\pi_{i}^{v c g}=\pi_{i}+\pi^{s}(p)-\pi^{s}\left(p_{-i}\right)$. This is true for every buyer $i \in B$. From Theorem 1, $p$ is a UCE price vector.

As noted earlier, the auctions in Ausubel and Milgrom [3] and de Vries et al. [11] are uQCE-invariant(0) auctions of the main economy. These auctions can be converted to a uQCE-invariant auction for the main economy (by modifying Step S2(0) to Step S2), and Theorem 4 shows that they terminate with VCG outcome under the BAS condition.

\subsection{Incentives}

Although the VCG mechanism is strategyproof and supports truthful bidding in a dominant strategy equilibrium, an ascending price auction that achieves the VCG outcome will not in general support truthful bidding in a dominant strategy equilibrium [16, 28, 3, 6, 11]. Instead, and with additional consistency requirements, then truthful bidding can be made an ex post Nash equilibrium for a uQCE-invariant auction.

Definition 11 In an ascending price auction, the bidding strategy of buyer $i$ is consistent if there is some general valuation profile $v^{\prime}$ for which the reported demand set $D_{i}\left(p^{t}\right)$ in each round $t$ satisfies

$$
D_{i}\left(p^{t}\right)=\left\{S \in \Omega: v_{i}^{\prime}(S)-p^{t}(S) \geq v_{i}^{\prime}(T)-p_{i}(T), \forall T \in \Omega\right\}
$$

In words, there is a valuation profile that explains the bidding strategy of a buyer as a truthful bidding strategy across all rounds of the auction. 
Consistency can be achieved in a uQCE-invariant auction through appropriate activity rules (e.g., see [4]) Denote the demand set submitted (possibly untruthfully) by buyer $i$ in round $t$ as $D_{i}\left(p^{t}\right)$ and consider the following activity rules (to be imposed in every round $t$ ):

- Round Monotonicity (RM): For every buyer $i \in B, D_{i}\left(p^{t}\right) \subseteq D_{i}\left(p^{t+1}\right)$.

- Bundle Monotonicity (BM): For every buyer $i \in B$, if $S \subseteq T$ and $S \in D_{i}\left(p^{t}\right)$, then $T \in D_{i}\left(p^{t}\right)$.

Under truthful bidding, round monotonicity is satisfied by Theorem 3. Bundle monotonicity is satisfied under truthful bidding in the first round by free disposal and in subsequent rounds by round monotonicity. We provide the proof of the sufficiency of the activity rules in the Appendix. ${ }^{10}$

Proposition 5 Under activity rules $R M$ and BM, every bidding strategy of buyers is consistent in uQCE-invariant auctions.

If we assume such consistency, then we have the following result. The proof resembles similar proofs in $[16,6,11]$ and is provided in the Appendix.

Theorem 5 In a uQCE-invariant auction with activity rules that ensure consistency, truthful bidding is an ex post Nash equilibrium.

This equilibrium does not require that the auction terminates with UCE prices for every valuation profile, as long as the auction terminates with $\mathrm{CE}$ prices of the main economy for any deviation from truthful bidding. This is useful for auctions that terminate with UCE prices for restricted valuations, but for which a deviation from truthful bidding may lead to termination with CE prices but not UCE prices, e.g., auctions in $[3,11]$.

This gives us a corollary for a uQCE-invariant auction for the main economy which achieves the VCG outcome only in restricted valuation domains. A sufficient condition for the existence of such an auction is the BAS condition (Theorem 4).

Corollary 1 If buyers are substitutes, then every uQCE-invariant auction for the main economy with activity rules that ensure consistency has truthful bidding as an ex post Nash equilibrium.

\subsection{Discussion}

In adopting different definitions for "adjusted buyers" we can define different uQCE-invariant auctions. In fact, one can select an arbitrary set of buyers $U^{t} \subseteq B^{+}\left(p^{t}\right)$ (i.e., who do not have $\emptyset$ in their demand sets and are still actively bidding) as the adjusted buyers. Such an auction will still maintain uQCE prices and terminate with the VCG outcome. This illustrates the power of our construction.

\footnotetext{
${ }^{10}$ There are other interesting ways to ensure such consistency in bidding (see for instance $[28,3]$ ).
} 
However, there is a drawback to selecting some arbitrary set with which to define price adjustments. On one hand, we prefer not to select the largest possible set of adjusted buyers in each round. The largest possible set is to simply increase prices on all buyers in $B^{+}\left(p^{t}\right)$. But, in this case we can unnecessarily increase the prices to some buyers. We prefer to avoid this because as prices approach the 'value=price' UCE price vector, our auctions elicit all the valuation information from buyer, and thus the auction loses this advantage over a sealed-bid auction. In addition, we prefer to keep the discounts as low as possible (and zero whenever possible) to improve the auction's transparency and simplicity. However, we prefer not to select the minimal set of adjusted buyers in each round. Such a set could be a single buyer in $B^{+}\left(p^{t}\right)$. Adjusting the price of one buyer at a time may lead to very slow progress of the auction particularly when additional necessary price adjustments are already known.

So, we prefer to strike a balance and adjust the prices on a set of buyers such that:

(i) we maintain transparency and avoid unecessary demand revelation by making only those price adjustments that are necessary to make progress towards UCE prices, and

(ii) we make quick enough progress in the auction by making multiple price adjustments in each round when there is evidence that prices to multiple buyers must be increased to make progress towards UCE prices.

In the rest of the paper, we will discuss two approaches to identify adjusted buyers in every round of a uQCE-invariant auction. These two approaches define two types of uQCEinvariant auctions which we relate to existing auctions in the literature. Besides adopting the economic properties outlined in this section, the specific auctions that we identify also have appeal to optimization algorithms (discussed in detail in [11]), and thus have a nice algorithmic justification.

\section{Adjusted Buyers via a Primal-Dual Algorithm}

In finding our first class of adjusted buyers, we adopt the primal-dual linear programming framework [5, 6, 11, 12]. We depart from previous work in one significant way. All the previous work considered primal-dual algorithms on the main economy and designed uQCEinvariant(0) auctions for the main economy. In contrast, we consider the main economy and every marginal economy simultaneously and formulate a primal problem to search for UCE prices. From this, we can find a class of adjusted buyers and thus a uQCE-invariant auction. To summarize the methodology:

- For every $M \in \mathbb{B}$, for economy $E(M)$, formulate the efficient allocation problem as a linear program and construct a restricted primal problem.

- Construct a single restricted primal problem by combining constraints from restricted primal problems for economy $E(M)$ for every $M \in \mathbb{B}$. 
- An optimal solution to the dual of this restricted primal gives us a set of adjusted buyers.

This yields a design for a uQCE-invariant auction that searches for an optimal primal and dual solution in economy $E(M)$ for every $M \in \mathbb{B}$ and thus finds a UCE price vector. ${ }^{11}$ We call this auction the primal-dual uQCE-invariant auction.

In defining the auction, we choose to omit the technical details of its interpretation as a primal-dual algorithm. The process of showing that such a price adjustment is a primaldual algorithm resembles the interpretations in earlier works [11, 6], and so we will defer these details to the Appendix. Instead, we will simply define the method used to define the adjusted buyers, which pins down the price-adjustment method and thus the particular auction instance in the class.

\subsection{The Primal-Dual uQCE-Invariant Auction}

To define our first auction, we first introduce some additional concepts. In general, an ascending price auction starts from a low price vector, where there is excess demand. The price vector in the auction is adjusted till there is no excess demand. In a uQCE-invariant auction, we adjust the price vector till there is no excess demand in the main and every marginal economy. Thus, uQCE prices are maintained in every round of the auction and the auction terminates with UCE prices. This idea of "universal" excess demand is used to define our adjusted buyers.

In general, our auction maintains a price vector $p \in \mathbb{R}_{+}^{|B| \times|\Omega|}$. Consider an economy $E(M)$ for some $M \in \mathbb{B}$. For any $X \in L^{*}\left(p_{M}\right)$ at price vector $p$, we say a buyer $i$ is unsatisfied in economy $E(M)$ if $X_{i} \notin D_{i}(p)$. Denote the total set of unsatisfied buyers in a set of buyers $K \subseteq M^{+}(p)$ at price vector $p$ in allocation $X \in L^{*}\left(p_{M}\right)$ as $\rho\left(X, K, p_{M}\right)$. Find an allocation in $X^{*} \in L^{*}\left(p_{M}\right)$ at price vector $p$ such that $\left|\rho\left(X^{*}, K, p_{M}\right)\right| \leq\left|\rho\left(X, K, p_{M}\right)\right|$ for every $X \in L^{*}\left(p_{M}\right)$. For simplicity, denote such a group of buyers as $\rho\left(K, p_{M}\right)$.

Definition 12 (Universally Undersupplied Buyers) ${ }^{12}$ A set of buyers $K \subseteq M^{+}(p)$ are undersupplied in economy $E(M)$ for some $M \in \mathbb{B}$ at price vector $p$ if $\left|\rho\left(K, p_{M}\right)\right|>0$ and $p_{M}$ is a QCE price vector of economy $E(M)$.

$A$ set of buyers $K \subseteq B^{+}(p)$ are universally undersupplied at price vector $p$ if buyers in $K \cap M^{+}(p)$ are undersupplied in economy $E(M)$ for some $M \in \mathbb{B}$ at price vector $p$ and $p$ is a $u$-QCE price vector.

\footnotetext{
${ }^{11}$ To reiterate, the auction is not searching for a solution to all $m+1$ economies using $m+1$ separate primaldual algorithms. Rather, the auction is a single primal-dual algorithm, that solves the efficient allocation problem of economy $E(M)$ for every $M \in \mathbb{B}$ simultaneously. Each buyer faces a single ascending price path.

${ }^{12}$ The idea of undersupplied buyers was introduced in de Vries et al. [11] and we generalize it to define universally undersupplied buyers.
} 
A set of buyers $K \subseteq B^{+}(p)$ are minimally universally undersupplied at price vector $p$ if there is no smaller $K^{\prime} \subsetneq K$ such that buyers in $K^{\prime}$ are also universally undersupplied at price vector $p$ and $p$ is a $u-Q C E$ price vector.

If a set of buyers $K$ are universally undersupplied at price vector $p$, then by definition $p$ is a uQCE price vector but not a UCE price vector. Thus, the buyers in $K$ cannot be satisfied simultaneously in any solution to winner determination in at least one economy $E(M)$, for some $M \in \mathbb{B}$.

Definition 13 (Price Adjustment Rule I) The adjusted buyers for the primal-dual $\boldsymbol{u Q C E}$-invariant auction in round $t$ is a set of minimally universally undersupplied buyers at price vector $p^{t}$.

One way to determine this adjusted buyers is to solve the traditional problem of winner determination in the following modified way:

(i) Solutions to winner determination are considered for economy $E(M)$, for every $M \in \mathbb{B}$.

(ii) For every $M \in \mathbb{B}$ and for every $K \subseteq B^{+}(p)$ at price $p$ in the auction, solutions to the winner determination problem of economy $E(M)$ is picked to minimize the number of unsatisfied buyers in the set of buyers $K$.

From Theorems 3 and 5, we immediately give the following corollary.

Corollary 2 The primal-dual uQCE-invariant auction achieves the VCG outcome for general valuations and truthful bidding is an ex post Nash equilibrium.

The primal-dual uQCE-invariant auction generalizes the primal-dual auction in de Vries et al. [11]. The adjusted buyers in round $t$ in the auction in de Vries et al. are a set of buyers which are minimally undersupplied in the main economy at price vector $p^{t}$. In our auction, we look for minimally universally undersupplied buyers for price adjustment.

Observe that if $K \subseteq B^{+}(p)$ is a set of minimally universally undersupplied buyers at price vector $p$, then $K$ is minimally undersupplied in economy $E(M)$ for some $M \in \mathbb{B}$ at price vector $p$. The approach of considering all economies simultaneously, using universally undersupplied buyers, is appealing because it gives us a natural way to select an economy $E(M)$ from which a set of minimally undersupplied buyers are chosen. Besides, it has a primal-dual algorithm interpretation that considers the main economy and every marginal economy simultaneously (see Appendix).

One can also adopt a sequential approach, which generalizes the primal-dual auction in de Vries et al. [11]. For this, at any round of the auction, we just pick some economy $E(M)$ for which a $\mathrm{CE}$ is not achieved yet and run one round of this earlier auction design on that marginal economy from the current prices. As noted above, de Vries et al. [11] find a set of minimally undersupplied buyers in order to adjust prices. In particular, in this sequential approach we can order the elements in $\mathbb{B}$ in some order and run this earlier auction for every element with each new stage starting from the closing prices of the last. If the main economy, $E(B)$, is the first element chosen, then in the first stage we run the exact auction in de Vries et al. [11]. 


\subsection{A Clinching Interpretation}

We will provide an alternative method to calculate the final discount in the primal-dual uQCE-invariant auction. This method determines the final discount dynamically during the auction and thus improves the transparency of the auction. This interpretation of the auction highlights some similarities with the earlier clinching and credit/debit auctions of Ausubel $[2,1]$.

For this, we will first define some concepts. At any round $t$ of the auction, let $K^{t}$ be the minimally universally undersupplied set of buyers. We will say a buyer $i \in K^{t}$ is satisfied in economy $E(M)$ in round $t$ if $i \notin \rho\left(K^{t}, p_{M}^{t}\right)$. Define $Q\left(p_{M}^{t}\right):=K^{t} \backslash \rho\left(K^{t}, p_{M}^{t}\right)$. Informally, $Q\left(p_{M}^{t}\right)$ is the set of all buyers who are satisfied and belong to $K^{t}$ (i.e., see a price increase) in economy $E(M)$ in round $t$. For simplicity, we will write the set of satisfied buyers in the main economy in round $t$ who see a price increase as $Q\left(p^{t}\right)$. Now, we can track the revenue of the seller across rounds.

Proposition 6 The change in revenue of the seller in economy $E(M)$ in a round $t$ of the primal-dual uQCE-invariant auction is $\left|Q\left(p_{M}^{t}\right)\right|$ for every $M \in \mathbb{B}$.

Proof: The proof proceeds in two steps.

Step 1: Consider economy $E(M)$ for any $M \in \mathbb{B}$ and let $K^{t}$ be a minimally universally undersupplied set of buyers in round $t$. So, we can satisfy any proper subset of buyers of $K^{t} \cap$ $M^{+}\left(p^{t}\right)$ simultaneously, i.e., for every $K^{\prime} \subsetneq K^{t}, \rho\left(K^{\prime}, p_{M}^{t}\right)=\emptyset$. Consider $T \subsetneq\left(K^{t} \cap M^{+}\left(p^{t}\right)\right)$ such that $T=\left(K^{t} \cap M^{+}\left(p^{t}\right)\right) \backslash\{i\}$. Since $K^{t}$ is minimally universally undersupplied, there exists some allocation $X \in L^{*}\left(p_{M}^{t}\right)$ such that for every $j \in T, X_{j} \in D_{j}\left(p^{t}\right)$. By considering $X$ again for simultaneously satisfying buyers in $K^{t}$, we get $\rho\left(K^{t}, p_{M}^{t}\right) \leq 1$ (the only potential buyer who cannot be satisfied is $i$ ). This means, $\left|Q\left(p_{M}^{t}\right)\right| \geq\left|K^{t} \cap M^{+}\left(p^{t}\right)\right|-1$. From the definition of $Q\left(p_{M}^{t}\right)$, we have $Q\left(p_{M}^{t}\right) \subseteq K^{t} \cap M^{+}\left(p^{t}\right)$. This gives us $\left|K^{t} \cap M^{+}\left(p^{t}\right)\right| \geq$ $\left|Q\left(p_{M}^{t}\right)\right| \geq\left|K^{t} \cap M^{+}\left(p^{t}\right)\right|-1$.

Step 2: Again, consider economy $E(M)$ for any $M \in \mathbb{B}$ and let $K^{t}$ be a minimally universally undersupplied set of buyers in round $t$. Let $X^{*} \in L^{*}\left(p_{M}^{t}\right)$ be an allocation such that $\rho\left(K^{t}, p_{M}^{t}\right)=\rho\left(X^{*}, K^{t}, p_{M}^{t}\right)$. Since $Q\left(p_{M}^{t}\right)$ denotes the set of satisfied buyers from $K^{t} \cap M^{+}\left(p^{t}\right)$ in economy $E(M)$, the revenue of the seller from $X^{*}$ is increased by $\left|Q\left(p_{M}^{t}\right)\right|$ due to price increase. Now, consider any other feasible allocation $X \neq X^{*}$ of economy $E(M)$. If $X \notin L\left(p_{M}^{t}\right)$, then the revenue from $X$ can increase by a maximum $\left|K^{t} \cap M^{+}\left(p^{t}\right)\right|$. If $X \in L\left(p_{M}^{t+1}\right)$, then the change in revenue of the seller in economy $E(M)$ becomes $\leq$ $\left|K^{t} \cap M^{+}\left(p^{t}\right)\right|-1 \leq\left|Q\left(p_{M}^{t}\right)\right|$ (using the result in Step 1). If $X \in L\left(p_{M}^{t}\right)$, the maximum number of buyers that can be satisfied in $K \cap M^{+}\left(p^{t}\right)$ is $\left|Q\left(p_{M}^{t}\right)\right|$ (using the result in Step 1). This means, the revenue from allocation $X$ can increase by a maximum of $\left|Q\left(p_{M}^{t}\right)\right|$. These arguments imply that the change in revenue of the seller is $\left|Q\left(p_{M}^{t}\right)\right|$ in economy $E(M)$.

Proposition 6 shows that by identifying the adjusted buyers we can also determine the change in revenue of economy $E(M)$ for every $M \in \mathbb{B}$ in a round of the primal-dual uQCE- 
invariant auction. ${ }^{13}$ This fact enables us to improve the transparency and simplicity of our auction by allowing us to calculate the discounts of the buyers dynamically.

In this modified version of the primal-dual uQCE-invariant auction, we maintain a vector of discounts on buyers in each round, starting from zero. In every round $t$ of the auction, the discount of buyer $i$ is updated by $\left|Q\left(p^{t}\right)\right|-\left|Q\left(p_{-i}^{t}\right)\right|$. From Proposition 6 , this is the difference between the increase in revenue in the main economy and the increase in revenue in the marginal economy without buyer $i$.

It is important to observe that for any buyer $i$, the discount (henceforth, denoted by $\kappa_{i}$ ) can increase or decrease or remain the same across rounds of the auction. For instance, consider a scenario when the minimally universally undersupplied set is a singleton set $\{k\}$. If $k$ is not in any of the revenue maximizing allocations of the main economy, then the revenue of the main economy will not increase. But $k$ can be in the revenue maximizing allocation of a marginal economy. This can increase the revenue of that marginal economy and thus decrease the discount of the corresponding buyer. An analogous analysis explains how the discount can increase in some rounds. If the value $\left|Q\left(p^{t}\right)\right|-\left|Q\left(p_{-i}^{t}\right)\right|$ is positive (negative), then it indicates crediting (debiting) to the price in a particular round. This is illustrated with an example in Table 2 in Section 4.3. Thus, we have both crediting and debiting. We call this variant of the primal-dual uQCE-invariant auction the primal-dual credit-debit auction. We give a formal definition below.

Definition 14 (Primal-Dual Credit-Debit Auction) The primal-dual credit-debit auction is defined as follows:

S0 The auction starts at the zero price vector. Set the initial discounts of buyers to zero: $\kappa_{i}=0$ for all $i \in B$.

S1 In round $t$ of the auction, with price vector $p^{t}$ :

S1.1 Collect demand sets of buyers at price vector $p^{t}$.

S1.2 If $p^{t}$ is a UCE price vector, then go to Step S2.

S1.3 Else, select a set of minimally universally undersupplied buyers $K^{t} \subseteq B^{+}\left(p^{t}\right)$ who see a price increase.

S1.3 If $i \in K^{t}$ and $S \in D_{i}\left(p^{t}\right)$, then $p_{i}^{t+1}(S):=p_{i}^{t}(S)+1$. Else, $p_{i}^{t+1}(S):=p_{i}^{t}(S)$.

$S 1.4$ For every buyer $i \in B$, update the discount $\kappa_{i}$ as $\kappa_{i}:=\kappa_{i}+\left[\left|Q\left(p^{t}\right)\right|-\left|Q\left(p_{-i}^{t}\right)\right|\right]$. Repeat from Step S1.1.

S2 The auction ends with the final allocation of the auction being any $X \in L^{*}(p)$ and final payment of every buyer $i \in B$ being $p_{i}\left(X_{i}\right)-\kappa_{i}$, where $p$ is the final price vector of the auction.

\footnotetext{
${ }^{13}$ We show in the Appendix that the minimally universally undersupplied buyers is the solution to a linear program and the change in revenue of every economy in a round can be found from the solution of the same linear program.
} 
Using Theorems 3 and 5, we immediately get the following corollary.

Corollary 3 The primal-dual credit-debit auction achieves the VCG outcome for general valuations and truthful bidding is an ex post Nash equilibrium.

It is interesting to compare this clinching and unclinching auction with that due to Ausubel [1], for the case of substitutes valuations, and from which we adopt our terminology. Ausubel also calculates the Vickrey payments dynamically (during the auction) through the use of "crediting" and "debiting" payments. Thus, the prices in both the primal-dual creditdebit auction and Ausubel's auction do not reflect the actual payment of buyers, rather the payment information of buyers is calculated dynamically as an adjustment to the price. However, Ausubel's auction is not a uQCE-invariant auction because the auction does not maintain a single price path but rather each buyers faces multiple price paths. There is no single final price vector that corresponds to a UCE price vector. Multiple price paths also allow Ausubel to adopt linear and anonymous prices for each path. We already established the impossibility of such simple prices within the class of auctions that we study, even for substitutes valuations.

\subsection{An Example}

Consider the example in Table 1 (b) in which the BAS condition fails. The progress of the primal-dual credit-debit auction in this example is illustrated in Table 2. The columns corresponding to buyers show prices on bundles. The bundles which have prices in $(\cdot)$ are in the demand set of the respective buyers. The seller's revenue in economy $E(M)$ for $M \in\left\{B, B_{-1}, B_{-2}, B_{-3}\right\}$ is shown in every round. In the last column, we show the update to the discounts of each buyer. Observe that the discount of each buyer (weakly) increases in every round except from round 3 to round 4 . The auction ends at a UCE price vector in 8 rounds.

\section{$5 \quad$ Extending the $i$ Bundle Auction}

In Parkes [24] (also see Parkes and Ungar [27]), an auction called $i$ Bundle was designed. The auction is also designed within a linear programming framework, but is distinguished from the auction of de Vries et al. [11] in that the price adjustment step in each round is that of a subgradient algorithm rather than a primal-dual algorithm. $i$ Bundle, like the auction of de Vries et al. [11], is an uQCE-invariant(0) auction for the main economy.

In this section, we extend $i$ Bundle to define a uQCE-invariant auction. We call this generalization $i$ Bundle, Extend and Adjust ( $i \mathrm{BEA}$ ). The price adjustment step in this auction is simpler than in the primal-dual uQCE-invariant auction. Also, the natural perception about auctions in practice is that losing buyers bid to increase the prices. This is the case in $i \mathrm{BEA}$, 


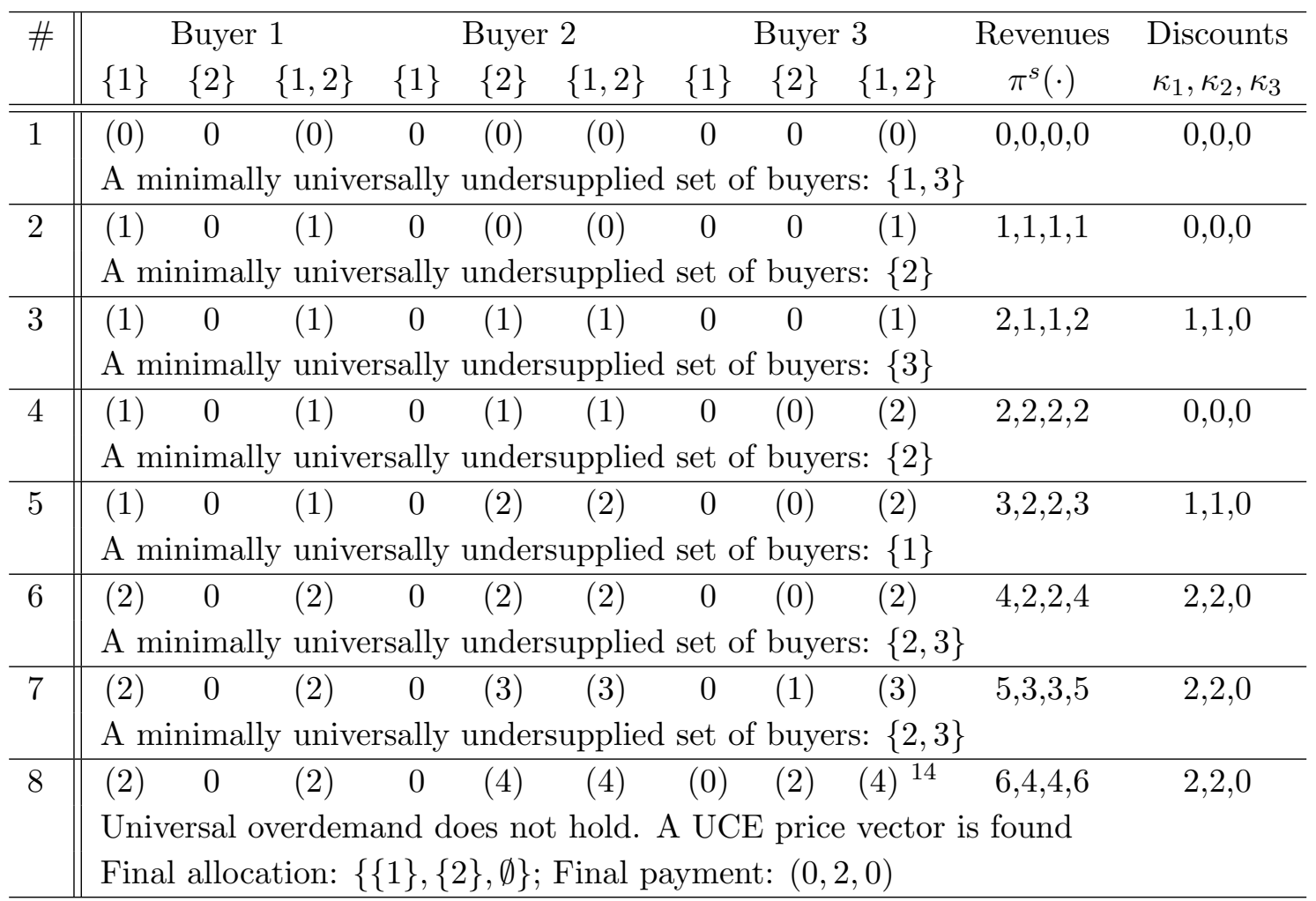

Table 2: Progress of the primal-dual credit-debit auction in an example for which buyers are not substitutes.

in which the adjusted buyers in each round are a set of losing buyers of some economy but is not the case in the primal-dual uQCE-invariant auction.

To describe $i$ Bundle, we reiterate that $\rho\left(M, p_{M}\right)$ denotes a minimum set of buyers from $M$ who cannot be satisfied at price $p_{M}$ in any allocation in $L^{*}\left(p_{M}\right)$. $i$ Bundle chooses such a set of buyers as the adjusted buyers. $i$ Bundle terminates as soon as $\mathrm{CE}$ prices of the main economy is reached and with no discount to buyers from the final prices.

To define $i$ BEA as a uQCE-invariant auction, we need to define the adjusted buyers for this auction:

Definition 15 (Price Adjustment Rule II) The adjusted buyers for $i$ BEA are found in round $t$ as follows:

- Select an economy $E(M)(M \in \mathbb{B})$ for which $\rho\left(M, p_{M}^{t}\right) \neq \emptyset$. Then $\rho\left(M, p_{M}^{t}\right)$ is the set of adjusted buyers.

Call the selected economy the pivot economy. In this definition, an economy that is not yet in $\mathrm{CE}$ is selected at random in each round and prices of a minimal set of unsatisfied buyers in that economy are adjusted. Using Theorems 3 and 5, the following corollary is immediate.

\footnotetext{
${ }^{14}$ Buyer 3 also demands $\emptyset$ in this round.
} 
Corollary 4 BEA achieves the VCG outcome for general valuations and truthful bidding is an ex post Nash equilibrium.

The following properties of uQCE-invariant auctions allow for a simplification to the definition of $i$ BEA.

Lemma 2 Consider a round $t$ of a $u Q C E$-invariant auction where the price vector $p^{t}$ is adjusted. If $p_{M}^{t}$ is a $C E$ price vector of economy $E(M)$ for some $M \in \mathbb{B}$, then $p_{M}^{t+1}$ is also a $C E$ price vector of economy $E(M)$.

Proof: Let $U^{t}$ be the set of adjusted buyers in round $t$ of the uQCE-invariant auction. By definition, $\left(U^{t} \cap M\right) \subseteq M^{+}\left(p^{t}\right)$. Since price increase in a uQCE-invariant auction is by unity, the maximum revenue increase of the seller is $\left|U^{t} \cap M\right|$. Let $\left(p_{M}^{t}, X\right)$ be a CE of economy $E(M)$. Since $\left(U^{t} \cap M\right) \subseteq M^{+}\left(p^{t}\right), X_{i} \in D_{i}\left(p^{t}\right)$ for every $i \in\left(U^{t} \cap M\right)$. By the price adjustment in uQCE-invariant auctions, the increase in revenue from allocation $X$ in economy $E(M)$ is $\left|\left(U^{t} \cap M\right)\right|$. This means, $X$ remains revenue maximizing for the seller at price vector $p_{M}^{t+1}$. From Lemma 1 , for every buyer $i \in M$, if $X_{i} \in D_{i}\left(p^{t}\right)$, then $X_{i} \in D_{i}\left(p^{t+1}\right)$. Thus, $\left(p_{M}^{t+1}, X\right)$ is a CE of economy $E(M)$.

The implication of Lemma 2 is that once we realize that a $\mathrm{CE}$ of economy $E(M)$ is achieved in $i \mathrm{BEA}$ we will not need to consider that economy again when selecting adjusted buyers in later rounds.

As with our first auction, we can again implement a "sequential" variation of $i$ BEA in which we order the economies defined in $\mathbb{B}$ in some sequence. Then, we consider these economies in that order one by one. For every set of buyers $M$ in that sequence, construct adjusted buyers as in Definition 15 in every round of $i \mathrm{BEA}$ until a $\mathrm{CE}$ of economy $E(M)$ is reached. If the main economy is the first such economy selected then buyers will know about their final allocation of items at the end of this phase. So, they may lose interest in participating in later rounds of the auction because bidding in these stages does not change their final allocation. By making the main economy the last pivot economy this issue can be overcome.

\subsection{A Clinching Interpretation of $i$ BEA}

The clinching interpretation idea for the primal-dual credit-debit auction can also be extended to $i \mathrm{BEA}$. At every round of $i \mathrm{BEA}$, for every buyer $i \in B$, we can define the new discount of $i$ as $\pi^{s}\left(p^{t}\right)-\pi^{s}\left(p_{-i}^{t}\right)$. With this, we can track the discounts of every buyer dynamically and apply it at the end of the auction. For an economy that has reached a CE, the increase in revenue can be calculated from the following lemma, proof of which follows from the arguments in the proof of Lemma 2.

Lemma 3 Consider a round $t$ of a uQCE-invariant auction where the price vector $p^{t}$ is adjusted. If $p^{t}$ is a $C E$ price vector of economy $E(M)$ for some $M \in \mathbb{B}$, then the increase in 
revenue of the seller in economy $E(M)$ is $\left|U^{t} \cap M\right|$, where $U^{t}$ is the set of adjusted buyers in round $t$.

The increase in revenue of an economy that has not yet reached a $\mathrm{CE}$ in $i \mathrm{BEA}$ is more difficult to track than in the earlier auction. Now, we must explicitly calculate the revenue in such an economy in each round, by solving an additional winner determination problem.

\subsection{An Example}

The example in Table 3 has three buyers and two items. The values of buyers are shown in the third row. Subsequent rows illustrate the progress of the auction in each round. Each row provides the prices on each bundle to each buyer, and the seller revenue in the main economy and in each marginal economy. The bid of each buyer is indicated with parentheses. Comments in each round indicate which allocation is selected to solve the winner determination (WD) problem. The main economy $E(B)$ is adopted as the initial pivot economy. $i$ Bundle for $E(B)$ terminates in round 7 , at which point the price vector is also a $\mathrm{CE}$ price vector of economies $E\left(B_{-2}\right)$ and $E\left(B_{-3}\right)$. Pivot economy $E\left(B_{-1}\right)$ is adopted for the final two rounds, at which point $i \mathrm{BEA}$ terminates with a UCE price vector.

\section{Summary and Open Questions}

We introduced a class of efficient ascending price combinatorial auctions. The auctions achieve the VCG outcome for private valuations that respect no externalities and free disposal, but without requiring any additional restrictions. Truthful bidding is an ex post Nash equilibrium for buyers. Crucially, we have relaxed the traditional definition of an ascending price auction to allow for discounts to buyers at the end of the auction. Our relaxation retains the simplicity of a single price path. The auctions use the concept of universal competitive equilibrium (UCE) prices. We showed that any ascending Vickrey auction for the combinatorial allocation problem must terminate with a UCE price vector, and that terminating with a UCE price vector is sufficient to determine the Vickrey payments.

We provided two specific examples of auctions within the general class. One of them generalizes the auction in de Vries et al. [11] from the restricted valuation class of buyersubmodularity (roughly equivalent to substitutes) to general valuations. The other generalizes the $i$ Bundle auction in Parkes and Ungar [27], also from the sub-class of buyersubmodularity to general valuations. The auctions can also be viewed as a generalization of Ausubel's clinching [2] and credit/debit auctions [1], because we provide interpretations for both auctions in which the final discounts of buyers are determined dynamically across rounds. Table 4 summarizes our contribution to the ascending price Vickrey auctions literature. Although the worst-case number of prices in our auctions is exponential in the number of items, this cost will generally not be incurred in practice. The number of prices that must be quoted is bounded by the number of bundles which interest the buyers. In special cases, 


\begin{tabular}{|c|c|c|c|c|}
\hline \multirow[b]{3}{*}{ Values } & Buyer 1 & Buyer 2 & Buyer 3 & \multirow{3}{*}{$\begin{array}{c}\text { Seller revenue } \\
\text { in main and } \\
\text { marginal economies }\end{array}$} \\
\hline & $\{1\} \quad\{2\} \quad\{1,2\}$ & $\{1\} \quad\{2\} \quad\{1,2\}$ & $\{1\} \quad\{2\} \quad\{1,2\}$ & \\
\hline & 3 & 6 & 2 & \\
\hline \multirow[t]{2}{*}{1} & $(0) \quad 0 \quad(0)$ & $\begin{array}{lll}0 & (0) & (0)\end{array}$ & $0 \quad(0)$ & $\{0,0,0,0\}$ \\
\hline & \multicolumn{4}{|c|}{ Pivot: $E(B)$. WD selects $\{\{1\},\{2\}, \emptyset\}$. Buyer $\{3\}$ is unsatisfied. } \\
\hline \multirow[t]{2}{*}{2} & $0 \quad(0)$ & $0 \quad(0)$ & $(1)$ & $\{1,1,1,0\}$ \\
\hline & \multicolumn{4}{|c|}{ Pivot: $E(B)$. WD selects $\{\emptyset, \emptyset,\{1,2\}\}$. Buyers $\{1,2\}$ are unsatisfied. } \\
\hline \multirow[t]{2}{*}{3} & $(1) \quad 0 \quad(1)$ & $0 \quad(1) \quad(1)$ & $0 \quad(1)$ & $\{2,1,1,2\}$ \\
\hline & \multicolumn{4}{|c|}{ Pivot: $E(B)$. WD selects $\{\{1\},\{2\}, \emptyset\}$. Buyer $\{3\}$ is unsatisfied. } \\
\hline \multirow[t]{2}{*}{4} & $0 \quad(1)$ & (1) & $0 \quad(0)$ & $\{2,2,2,2\}$ \\
\hline & \multicolumn{4}{|c|}{ Pivot: $E(B)$. WD selects $\{\{1\},\{2\}, \emptyset\}$. Buyer $\{3\}$ is unsatisfied. } \\
\hline \multirow[t]{2}{*}{5} & $0 \quad(1)$ & $(1)$ & $(1)$ & $\{3,3,3,2\}$ \\
\hline & \multicolumn{4}{|c|}{ Pivot: $E(B)$. WD selects $\{\emptyset, \emptyset,\{1,2\}\}$. Buyers $\{1,2\}$ are unsatisfied. } \\
\hline \multirow[t]{2}{*}{6} & $(2)$ & $(2) \quad(2)$ & $(0) \quad(1)$ & $\{4,3,3,4\}$ \\
\hline & \multicolumn{4}{|c|}{ Pivot: $E(B)$. WD selects $\{\{1\},\{2\}, \emptyset\}$. Buyer $\{3\}$ is unsatisfied. } \\
\hline \multirow[t]{4}{*}{7} & $(2)$ & $(2)$ & $\begin{array}{lll}(0) & (2) & (4)^{15}\end{array}$ & $\{4,4,4,4\}$ \\
\hline & \multirow{3}{*}{\multicolumn{4}{|c|}{$\begin{array}{l}\text { CEs of economies } E(B), E\left(B_{-2}\right), E\left(B_{-3}\right) \text { are reached. } \\
\{\{1\},\{2\}, \emptyset\} \text { is an efficient allocation of } E(B) \text {. } \\
\text { Pivot: } E\left(B_{-1}\right) \text {. WD selects }\{\emptyset, \emptyset,\{1,2\}\} \text {. Buyer }\{2\} \text { is unsatisfied. }\end{array}$}} \\
\hline & & & & \\
\hline & & & & \\
\hline \multirow[t]{2}{*}{8} & $(2) \quad 0 \quad(2)$ & $0 \quad(3) \quad(3)$ & $(0) \quad(2) \quad(4)$ & $\{5,4,4,5\}$ \\
\hline & \multicolumn{4}{|c|}{ Pivot: $E\left(B_{-1}\right)$. WD selects $\{\emptyset, \emptyset,\{1,2\}\}$. Buyer $\{2\}$ is unsatisfied. } \\
\hline \multirow[t]{4}{*}{9} & $(2) \quad 0 \quad(2)$ & $0 \quad(4) \quad(4)$ & $(0) \quad(2) \quad(4)$ & $\{6,4,4,6\}$ \\
\hline & \multicolumn{4}{|c|}{ An UCE price vector is reached. } \\
\hline & \multicolumn{4}{|c|}{ Final allocation: $\{\{1\},\{2\}, \emptyset\}$} \\
\hline & \multicolumn{4}{|c|}{ Final payment: $p_{1}(\{1\})=2-[6-4]=0, p_{2}(\{2\})=4-[6-4]=2, p_{3}(\emptyset)=0$} \\
\hline
\end{tabular}

Table 3: Progress of $i$ BEA for an example

like the homogeneous items/units case, the number of such bundles can be polynomial (in the number of units).

An interesting direction for future work is to find a characterization of valuations for which auctions with linear and non-anonymous prices or non-linear and anonymous prices can achieve the VCG outcome.

\footnotetext{
${ }^{15}$ Buyer 3 also demands $\emptyset$ bundle from this round onwards.
} 


\begin{tabular}{|c|c|c|c|c|}
\hline $\begin{array}{l}\text { Auctions } \\
\text {-Type of items- }\end{array}$ & $\begin{array}{l}\text { Conditions under } \\
\text { which the VCG } \\
\text { outcome is achieved }\end{array}$ & $\begin{array}{l}\text { Number of } \\
\text { price paths }\end{array}$ & $\begin{array}{l}\text { Search for } \\
\text { a CE of } \\
\text { economy }\end{array}$ & $\begin{array}{l}\text { Is final price } \\
\text { equal to final } \\
\text { payment? }\end{array}$ \\
\hline $\begin{array}{l}\text { Demange et al. [13] } \\
\text {-Heterogeneous- }\end{array}$ & Unit demand & Single & Main & Yes \\
\hline $\begin{array}{l}\text { Ausubel [1] } \\
\text {-Homogeneous- }\end{array}$ & $\begin{array}{l}\text { Non-increasing } \\
\text { marginal values }\end{array}$ & Single & Main & Yes \\
\hline $\begin{array}{l}\text { de Vries et al. [11] } \\
\text { Ausubel and Milgrom [3] } \\
\text {-Heterogeneous- }\end{array}$ & Buyers are submodular & Single & Main & Yes \\
\hline $\begin{array}{l}\text { de Vries et al. [11] } \\
\text { (Modified with } \\
\text { multiple price paths) } \\
\text {-Heterogeneous- }\end{array}$ & General valuations & Multiple & Main & $\begin{array}{l}\text { Yes } \\
\text { (but on } \\
\text { different } \\
\text { price paths) }\end{array}$ \\
\hline $\begin{array}{l}\text { Ausubel }[2] \\
\text {-Heterogeneous- }\end{array}$ & Gross substitutes & Multiple & $\begin{array}{l}\text { Main and } \\
\text { marginal }\end{array}$ & No \\
\hline
\end{tabular}

\section{Our Contribution}

\begin{tabular}{|l||l|l|l|l|}
\hline \hline $\begin{array}{l}\text { uQCE-invariant } \\
\text { auctions for } \\
\text { main economy } \\
\text {-Heterogeneous- }\end{array}$ & Buyers are substitutes & Single & Main & No \\
\hline $\begin{array}{l}\text { uQCE-invariant } \\
\text { auctions } \\
\text {-Heterogeneous- }\end{array}$ & General valuations & Single & $\begin{array}{l}\text { Main and } \\
\text { marginal }\end{array}$ & No \\
\hline
\end{tabular}

Table 4: Characteristics of Ascending Price Vickrey Auctions for Multiple Items

\section{References}

[1] Lawrence M. Ausubel. An Efficient Dynamic Auction for Heterogeneous Commodities. Working Paper, University of Maryland, July 2002.

[2] Lawrence M. Ausubel. An Efficient Ascending-Bid Auction for Multiple Objects. American Economic Review, 94(5):1452-1475, December 2004.

[3] Lawrence M. Ausubel and Paul R. Milgrom. Ascending Auctions with Package Bidding. Frontiers of Theoretical Economics, 1(1):1-42, 2002.

[4] Lawrence M. Ausubel and Paul R. Milgrom. Ascending Proxy Auctions. In Peter Cramton, Yoav Shoham, and Richard Steinberg, editors, Combinatorial Auctions (Chapter 3). MIT Press, 2005. Forthcoming.

[5] Sushil Bikhchandani, Sven de Vries, James Schummer, and Rakesh V. Vohra. Linear Programming and Vickrey Auctions. In Mathematics of the Internet: E-Auction and 
Markets, volume 127, pages 75-116. IMA Volumes in Mathematics and its Applications, September 2001.

[6] Sushil Bikhchandani and Joseph Ostroy. Ascending Price Vickrey Auctions. Forthcoming in Games and Economic Behavior, August 2002.

[7] Sushil Bikhchandani and Joseph Ostroy. The Package Assignment Model. Journal of Economic Theory, 107(2), December 2002.

[8] Edward Clarke. Multipart Pricing of Public Goods. Public Choice, 8:19-33, 1971.

[9] Olivier Compte and Philippe Jehiel. Auctions and information acquisition: Sealed-bid or Dynamic Formats? Technical report, CERAS and UCL, 2002.

[10] Peter Cramton. Ascending Auctions. European Economic Review, 42:745-756, 1998.

[11] Sven de Vries, James Schummer, and Rakesh V. Vohra. On Ascending Vickrey Auctions for Heterogeneous Objects. Working Paper, Kellogg School of Management, Northwestern University, 2005.

[12] Sven de Vries and Rakesh V. Vohra. Combinatorial Auctions: A Survey. Informs Journal on Computing, 15(3):284-309, 2003.

[13] Gabrielle Demange, David Gale, and Marilda Sotomayor. Multi-item Auctions. Journal of Political Economy, 94(4):863-872, 1986.

[14] Jerry R. Green and Jean-Jacques Laffont. Characterization of Satisfactory Mechanisms for the Revelation of Preferences for Public Goods. Econometrica, 45:427-438, 1977.

[15] Theodore Groves. Incentives in Teams. Econometrica, 41:617-631, 1973.

[16] Faruk Gul and Ennio Stacchetti. The English Auction with Differentiated Commodities. Journal of Economic Theory, 92(1):66-95, May 2000.

[17] Philippe Jehiel, Moritz Meyer ter Vehn, Benny Moldovanu, and William R. Zame. Limits of Ex-Post Implementation. Technical report, University of Bonn, 2005.

[18] Alexander S. Kelso and Vincent P. Crawford. Job Matching, Coalition Formation, and Gross Substitutes. Econometrica, 50(6):1483-1504, November 1982.

[19] Herman B. Leonard. Elicitation of Honest Preferences for the Assignment of Individuals to Positions. Journal of Political Economy, 91(3):461-479, June 1983.

[20] Debasis Mishra. Simple Primal-Dual Auctions are not Possible. In Proceedings of $5^{\text {th }}$ ACM Conference on Electronic Commerce (EC'04), New York City, NY, 2004. Appeared as a brief announcement. 
[21] Motty Perry and Philip J. Reny. An Efficient Multi-Unit Ascending Auction. Technical report, University of Chicago, 2004.

[22] Noam Nissan and Ilya Segal. The Communication Requirements of Efficient Allocations and Supporting Prices. Journal of Economic Theory, 2004. Forthcoming.

[23] Christos H. Papadimitriou and Kenneth Steiglitz. Combinatorial Optimization. Prentice Hall, Inc., Englewood Cliffs, NJ, 1982.

[24] David C. Parkes. ibundle: An Efficient Ascending Price Bundle Auction. In Proceedings of ACM Conference on Electronic Commerce (EC-99), Denver, CO, pages 148-157, 1999.

[25] David C. Parkes. Price-Based Information Certificates for Minimal-Revelation Combinatorial Auctions. In Julian Padget, David Parkes, Norman Sadeh, Onn Shehory, and William Walsh, editors, Agent Mediated Electronic Commerce IV: Designing Mechanisms and Systems (LNAI 2531), pages 148-157. Springer Verlag, 2002.

[26] David C. Parkes. Auction Design with Costly Preference Elicitation. Annals of Mathematics and AI, 2004. Special Issue on the Foundations of Electronic Commerce, Forthcoming.

[27] David C. Parkes and Lyle H. Ungar. Iterative Combinatorial Auctions: Theory and Practice. In Proceedings of the $17^{\text {th }}$ National Conference on Artificial Intelligence (AAAI-00), pages 74-81, 2000.

[28] David C. Parkes and Lyle H. Ungar. Preventing Strategic Manipulation in Iterative Auctions: Theory and Practice. In Proceeding of the 17th National Conference on Artificial Intelligence, (AAAI-00), pages 74-81, 2000.

[29] Stephen Rassenti, Vernon L. Smith, and Robert L. Bulfin. A Combinatorial Auction Mechanism for Airport Time Slot Allocation. Bell Journal of Economics, 12(2):402-417, 1982.

[30] Michael H. Rothkopf, Aleksander Pekec, and Ronald M. Harstad. Computationally Manageable Combinatorial Auctions. Management Science, 44(8):1131-1147, 1998.

[31] William Vickrey. Counterspeculation, Auctions, and Competitive Sealed Tenders. Journal of Finance, 16:8-37, 1961. 


\section{Appendix: The Primal-Dual uQCE-Invariant Auction}

In this section, we interpret our primal-dual uQCE-invariant auction as a primal-dual algorithm. ${ }^{16}$ We provide no new results in this section, but give an alternate (algorithmic) view of the primal-dual uQCE-invariant auction defined in Section 4.

To do this, we formulate the problem of finding a UCE price vector as a linear program. Using primal and dual feasibility with CS conditions CS-1 and CS-2, we formulate the CE of economy $E(M)$ for any $M \subseteq B$ as follows $\left(y_{i}^{M}(S)=1\right.$ denotes the allocation of bundle $S$ to buyer $i, z^{M}(X)=1$ denotes that the seller has selected allocation $X$, and the superscripts indicate that the variables corresponding to economy $E(M))$ :

$$
\begin{array}{rlrl}
y_{i}^{M}(S) & =\sum_{X \in L^{*}\left(p_{M}\right): X_{i}=S} z^{M}(X) \quad \forall i \in M, \forall S \in D_{i}(p) \cup\{\emptyset\} . \\
\sum_{S \in D_{i}(p)} y_{i}^{M}(S) & =1 \quad \forall i \in M^{+}(p) . \\
\sum_{\emptyset \neq S \in D_{i}(p)} y_{i}^{M}(S) \leq 1 & & \forall i \in M \backslash M^{+}(p) . \\
\sum_{X \in L^{*}\left(p_{M}\right)} z^{M}(X) & =1 . \\
y_{i}^{M}(S) \geq 0 & & \\
z^{M}(X) \geq 0 & \forall i \in M, \forall S \in D_{i}(p) \cup\{\emptyset\} .
\end{array}
$$

$(\mathbf{C E}(M, p))$

The first set of constraints in (5) enforce the balance of supply and demand. Constraints (6) and (7) indicate the demand in the economy, with buyers assigned to bundles in their demand set only. Constraint (8) indicates the supply, with a seller selecting an allocation that maximizes his payoff and is compatible with the demands of the buyers. Any allocation in $L^{*}\left(p_{M}\right)$ is such an allocation, by definition. If the linear program is feasible at price vector $p$, then $p_{M}$ is a CE price vector of economy $E(M)$. If the linear program is feasible at price vector $p$ for every $M \in \mathbb{B}$, then $p$ is also a $\mathrm{UCE}$ price vector.

In the spirit of a primal-dual algorithm, we can construct a restricted primal problem for formulation $(\mathbf{P}(M))$. This is already achieved in de Vries et al. [11], who adopt a primal-dual algorithm for the main economy to design their auction. In our formulation, we combine the restricted primal problems of the main economy and every marginal economy and maintain a single price vector (a set of dual variables) for the main and the marginal economies. This yields a uQCE-invariant auction.

Formally, our restricted primal is introduced by relaxing the $\mathrm{CE}$ formulation $(\mathbf{C E}(M, p))$ by introducing artificial variables $\left(\delta^{M}\right)$ into constraints (6) for every $M \in \mathbb{B}$. Using this, we

\footnotetext{
${ }^{16}$ Papadimitriou and Steiglitz [23] provide a useful text-book treatment of the family of primal-dual algorithms.
} 
define the quasi-CE price vector in an alternative manner using a set of linear inequalities:

Definition 16 (Quasi-CE Price) A price vector $p$ is a quasi-CE price vector of economy $E(M)$ for some $M \subseteq B$, if there is a feasible solution to $(\boldsymbol{C E}(M, p))$ with constraints (6) relaxed to:

$$
\begin{aligned}
\sum_{S \in D_{i}(p)} y_{i}^{M}(S)+\delta_{i}^{M}=1 & \forall i \in M^{+}(p) \\
\delta_{i}^{M} \geq 0 & \forall i \in M^{+}(p) .
\end{aligned}
$$

The effect of the relaxation is to allow the seller to still select a revenue maximizing allocation from $L^{*}\left(p_{M}\right)$, but to relax constraints (6) so that having buyers who are not allocated a bundle from their respective demand sets can be allowed. In particular, in a solution for which all artificial variables $\left(\delta_{i}^{M}\right)$ are zero, $p$ is a CE price vector of economy $E(M)$, and if such feasible solutions exist for every $M \in \mathbb{B}$ then $p$ is also a UCE price vector.

The relaxed formulation is useful because it is always feasible as long as $L^{*}\left(p_{M}\right)$ is nonempty (since values for variables $y^{M}, z^{M}, \delta^{M}$ can then be chosen to attain feasibility). Now, denote the feasible region of $(\mathbf{Q U A S I}(M, p))$ at price vector $p$ as $F(M, p)$. Our restricted primal problem introduces these artificial variables for economy $E(M)$ for every $M \in \mathbb{B}$ and tries to minimize their summed value. Combining the feasible regions of $(\mathbf{Q U A S I}(M, p))$ in economies $E(M)$ for every $M \in \mathbb{B}$, we can write the following restricted primal for any $K \subseteq B^{+}(p):$

$$
\begin{aligned}
\Delta(K, p) & =\max -\sum_{M \in \mathbb{B}} \sum_{i \in K \cap M^{+}(p)} \delta_{i}^{M} . \\
\text { s.t. }\left(y^{M}, z^{M}, \delta^{M}\right) & \in F(M, p) \quad \forall M \in \mathbb{B} .
\end{aligned}
$$

If buyer $i$ has $\delta_{i}^{M}=0$ for all $M \in \mathbb{B}$, we call $i$ a satisfied buyer. Else, $i$ is called an unsatisfied buyer.

The feasible region of $(\mathbf{R P}(K, p))$ is the union of feasible regions of $(\mathbf{Q U A S I}(M, p))$ for all $M \in \mathbb{B}$. For any $K \in B^{+}(p)$, the value of the objective function indicates the number of buyers from $K$ that cannot be satisfied simultaneously in the main economy as well as in every marginal economy. Formulation $(\mathbf{R P}(K, p))$ is feasible if $p$ a uQCE price vector. Observe that the feasible region of $(\mathbf{R P}(K, p))$ is independent of the choice of $K \subseteq B^{+}(p)$.

Any uQCE-invariant auction will start with a feasible solution to $(\mathbf{R P}(K, p))$, and identify adjusted buyers to maintain a uQCE price vector. Observe that if $\Delta(K, p)<0$, then for some $M \subseteq B, K$ is a set of undersupplied buyers in economy $E(M)$. This gives us an alternate definition of universally undersupplied buyers, which is useful to interpret our auction as a primal-dual algorithm.

Definition 17 (Universally Undersupplied Buyers) A set of buyers $K \subseteq B^{+}(p)$ is universally undersupplied at price vector $p$ if $p$ is a $u Q C E$ price vector and $\Delta(K, p)<0$. 
Given a universally undersupplied set of buyers $K$, it is sufficient that $\Delta\left(K_{-i}, p\right)=0$ for every $i \in K$ for $K$ to be minimally universally undersupplied. This can be seen from the fact that the feasible region of $(\mathbf{R P}(K, p))$ is independent of $K$. Thus, at any price vector $p$, a feasible solution of $\left(\mathbf{R P}\left(K_{-i}, p\right)\right)$ is also feasible for $\mathbf{R P}\left(K^{\prime}, p\right)$ for every $K^{\prime} \subseteq K_{-i}$. Now, consider the optimal solution of $\left(\mathbf{R P}\left(K_{-i}, p\right)\right)$ at price vector $p$ for which $\Delta\left(K_{-i}, p\right)=0$. For any $K^{\prime} \subseteq K_{-i}$, this solution is also feasible for $\left(\mathbf{R P}\left(K^{\prime}, p\right)\right)$ and $\Delta\left(K^{\prime}, p\right)=0$.

Given the restricted primal formulation we can proceed to define our adjusted buyers. If the optimal solution to $\left(\mathbf{R P}\left(B^{+}(p), p\right)\right)$ is zero, we have found a UCE price vector and we stop. Else, we look for the dual of $(\mathbf{R P}(K, p))$ for some universally undersupplied buyers $K \subseteq B^{+}(p)$ to define a class of adjusted buyers.

We note that the primal problem for which we are solving the primal-dual algorithm is simply the efficient allocation problem of the main economy and every marginal economies combined together. So, the dual of such a primal problem will have a separate price variable (and payoff variables for the buyers and the seller) for every economy. Thus, the dual of our restricted primal should give different price adjustment directions for each of these price vectors. But, what we show is that the same price adjustment (and thus payoff adjustments) is made across all economies. This is possible because of the starting dual feasible solution we take and the way we do price adjustment. Thus, and starting from a single price vector for all economies, our price adjustment direction allows us to maintain a single price vector for the main and for every marginal economy throughout the auction.

At any price vector $p$, if $\Delta\left(B^{+}(p), p\right)=0$, then the auction (primal-dual algorithm) can terminate. Otherwise, we find an optimal solution to the dual of $(\mathbf{R P}(K, p))$, for some $K \subseteq B^{+}(p)$ for which $\Delta(K, p)<0$, which is the following formulation:

$$
\Delta(K, p)=\min \sum_{M \in \mathbb{B}}\left[\lambda^{M}+\sum_{i \in M} \theta_{i}^{M}\right] .
$$

s.t.

$$
\begin{aligned}
\theta_{i}^{M}+\rho_{i}^{M}(S) & \geq 0 & & \forall M \in \mathbb{B}, \forall i \in M, \forall S \in D_{i}(p) . \\
\lambda^{M}-\rho_{i}^{M}\left(X_{i}\right) & \geq 0 & & \forall M \in \mathbb{B}, \forall X \in L^{*}\left(p_{M}\right) . \\
\theta_{i}^{M} & \geq 0 & & \forall M \in \mathbb{B}, \forall i \in M \backslash\left(K \cap M^{+}(p)\right) . \\
\theta_{i}^{M} & \geq-1 & & \forall M \in \mathbb{B}, \forall i \in K \cap M^{+}(p) . \\
\rho_{i}^{M}(\emptyset) & \geq 0 & & \forall M \in \mathbb{B}, \forall i \in M^{+}(p),
\end{aligned}
$$

Variable $\lambda^{M}$ will be interpreted as the change in revenue of the seller in economy $E(M)$ in this round, and $\theta_{i}^{M}$ will be interpreted as the change in payoff of buyer $i$. An optimal solution to this problem provides the direction in which we adjust prices in the auction:

Lemma 4 If $p$ is a $u Q C E$ price vector, then for any universally undersupplied set of buyers $K \subseteq B^{+}(p)$, there is an optimal solution to $(\boldsymbol{D R P}(K, p))$ such that $\rho_{i}^{M}(S)=1 \forall M \in$ $\mathbb{B}, \forall i \in K \cap M^{+}(p), \forall S \in D_{i}(p)$ and $\rho_{i}^{M}(S)=0$ otherwise. 
Proof: For any $M \in \mathbb{B}$, we say a buyer $i \in M$ is satisfied in economy $E(M)$ if $\delta_{i}^{M}=0$ in the optimal solution of restricted primal $(\mathbf{R P}(K, p))$. Let $Q\left(p_{M}\right)$ denote the set of satisfied buyers from set $K \cap M^{+}(p)$ in economy $E(M)$ for every $M \in \mathbb{B}$ at price vector $p$. This means that at optimality of $(\mathbf{R P}(K, p))$ we have,

$$
\Delta(K, p)=\sum_{M \in \mathbb{B}}\left[\left|Q\left(p_{M}\right)\right|-\left|K \cap M^{+}(p)\right|\right] .
$$

Set $\theta_{i}^{M}=-1$ for all $i \in K \cap M^{+}(p)$ and $\theta_{i}^{M}=0$ otherwise for every $M \in \mathbb{B}$. Set $\lambda^{M}=\left|Q\left(p_{M}\right)\right|$ for every $M \in \mathbb{B}$. This is clearly feasible for $(\operatorname{DRP}(K, p))$ and optimal using Equation 9.

Lemma 4 shows that the particular set of adjusted buyers in the primal-dual uQCEinvariant auction is an optimal solution of the dual of an appropriate restricted primal and thus defines a primal-dual price adjustment direction. This gives an algorithmic justification for the price adjustment.

There is still some flexibility in exactly how to adjust prices, because Lemma 4 allows one to choose any set of universally undersupplied buyers on which to adjust prices. For instance, the largest universally undersupplied set of buyers is $B^{+}(p)$, but there can be smaller sets.

We choose to adjust the prices on a minimally universally undersupplied set of buyers for reasons explained earlier in Section 3.5. Thus, a minimally universally undersupplied set of buyers define a class of adjusted buyers. This in turn defines a uQCE-invariant auction using a primal-dual algorithm which is the primal-dual uQCE-invariant auction.

\section{Appendix: Some Proofs}

\section{Proof of Proposition 5}

Proof: Let $p$ be the price vector in the auction in some round. We will show that bidding strategy of every buyer is consistent in each round $t$ till this round. By bundle monotonicity, for every buyer $i \notin B^{+}(p)$, we have $D_{i}(p)=\Omega$. Also, by round monotonicity, if a bundle $S \notin D_{i}(p)$, then $p_{i}(S)=0$. Now, construct the valuation profile $v^{\epsilon}$ (using $p$ ) as follows. For every buyer $i \in B^{+}(p)$,

$$
v_{i}^{\epsilon}(S)= \begin{cases}p_{i}(S)+\epsilon & \forall S \in D_{i}(p) \\ p_{i}(S)=0 & \text { otherwise }\end{cases}
$$

for some small $\epsilon>0$. For every buyer $i \in B \backslash B^{+}(p)$, construct valuation, $v_{i}^{\epsilon}$, as

$$
v_{i}^{\epsilon}(S)=p_{i}(S) \forall S \in D_{i}(p)=\Omega
$$

Consider a buyer $i$ and any bundle $S \in \Omega$. If $S \in D_{i}(p)$, then by bundle monotonicity for every $T \supseteq S$, we have $T \in D_{i}(p)$. From, the starting price vector and round monotonicity, $p_{i}(T) \geq p_{i}(S)$. This implies $v_{i}(T) \geq v_{i}(S)$. If $S \notin D_{i}(p)$, by round monotonicity and 
starting price vector, we get $p_{i}(S)=0$ and thus $v_{i}(S)=0$. So, for every $T \supseteq S$, we have $v_{i}(T) \geq v_{i}(S)$. This shows that $v_{i}^{\epsilon}$ is a general valuation satisfying free disposal.

For every buyer $i \in B$, every bundle $S \in \Omega$ and every round $t$ of the auction, define $\delta_{i}^{t}(S):=p_{i}(S)-p_{i}^{t}(S)$. Also, observe the following:

1. If $S \in D_{i}\left(p^{t}\right)$, then $S \in D_{i}(p)$ (round monotonicity). This means, $\delta_{i}^{t}(S)=r-t$, where $r$ denotes the number of rounds in the auction so far. So, from the definition of $v_{i}^{\epsilon}$, the payoff of buyer $i$ from bundle $S$ is: $\epsilon+(r-t)$ if $i \in B^{+}(p)$ and $r-t$ if $i \notin B^{+}(p)$.

2. If $S \notin D_{i}\left(p^{t}\right)$, then $\delta_{i}^{t}(S)<r-t$. From the definition of $v_{i}^{\epsilon}$, the payoff of buyer $i$ from bundle $S$ is less than $r-t$.

These two observations show that in every round of the auction so far, $v^{\epsilon}$ is consistent with the demand set profile in that round.

\section{Proof of Theorem 5}

Proof: Assume that there is a buyer $i$ which does not follow truthful bidding, while other buyers follow truthful bidding. Buyer $i$ has no incentive to follow a strategy which makes the auction run infinitely. So, buyer $i$ is (weakly) better off following a strategy in which the auction terminates. Let $p$ be the final price vector in the auction. From Proposition 5, consistency is possible using activity rules RM and BM in every round of the auction.

Since bidding in every round is consistent with some valuation profile, every uQCEinvariant auction terminates finitely such that if $X$ is the final allocation chosen and $p$ is the final price vector in the auction, then $X_{i} \in D_{i}(p)$ for every $i \in B$ and the revenue of the seller in the main economy is given by $\pi^{s}(p)=\sum_{i \in B} p_{i}\left(X_{i}\right)$.

Now, assume that there is some buyer $i$ which does not follow truthful bidding strategy whereas buyers in $B_{-i}$ follow truthful bidding strategy. This means, $\left(p_{-i}, \pi_{-i}(p), \pi^{s}\left(p_{-i}\right)\right)$ is a dual feasible solution of $\left(\mathbf{D}\left(B_{-i}\right)\right)$ and, we can write the following from weak duality:

$$
\sum_{j \in B_{-i}} \pi_{j}(p)+\pi^{s}\left(p_{-i}\right) \geq V\left(B_{-i}\right)
$$

Let $Y$ be an efficient allocation of the main economy and $\pi_{i}^{v c g}$ be the Vickrey payoff of buyer 
$i$. Now,

$$
\begin{aligned}
\pi_{i}^{v c g} & =V(B)-V\left(B_{-i}\right) \\
& =v_{i}\left(Y_{i}\right)+\sum_{j \in B_{-i}} v_{j}\left(Y_{j}\right)-V\left(B_{-i}\right) \\
& \left.\geq v_{i}\left(X_{i}\right)+\sum_{j \in B_{-i}} v_{j}\left(X_{j}\right)-V\left(B_{-i}\right) \quad \text { (From efficiency of } Y\right) \\
& =v_{i}\left(X_{i}\right)-p_{i}\left(X_{i}\right)+\sum_{j \in B_{-i}}\left[v_{j}\left(X_{j}\right)-p_{j}\left(X_{j}\right)\right]+\sum_{j \in B} p_{j}\left(X_{j}\right)-V\left(B_{-i}\right) \\
& =v_{i}\left(X_{i}\right)-p_{i}\left(X_{i}\right)+\sum_{j \in B_{-i}} \pi_{j}(p)+\pi^{s}(p)-V\left(B_{-i}\right) \\
& \geq v_{i}\left(X_{i}\right)-p_{i}\left(X_{i}\right)+\pi^{s}(p)-\pi^{s}\left(p_{-i}\right) \quad(\text { From Equation }(10)) .
\end{aligned}
$$

But, by the payment rule of a uQCE-invariant auction, $v_{i}\left(X_{i}\right)-p_{i}\left(X_{i}\right)+\pi^{s}(p)-\pi^{s}\left(p_{-i}\right)$ is the actual payoff (after discount) of buyer $i$ at the end of such an auction. This means, buyer $i$ is better off following the truthful bidding strategy. 